\title{
Ureadepsipeptides as ClpP Activators
}

Elizabeth C. Griffith, ${ }^{1+}$ Ying Zhao,${ }^{1+}$ Aman P. Singh, ${ }^{1}$ Brian P. Conlon, ${ }^{2}$ Rajendra Tangallapally, ${ }^{1}$ William R. Shadrick, ${ }^{1,}{ }^{\ddagger}$ Jiuyu Liu, ${ }^{1}$ Miranda J. Wallace, ${ }^{1}$ Lei Yang, ${ }^{1}$ John M. Elmore,${ }^{1}$ Yong Li, ${ }^{1}$ Zhong Zheng, ${ }^{1}$ Darcie J. Miller, ${ }^{3}$ Martin N. Cheramie, ${ }^{1}$ Robin B. Lee, ${ }^{1}$ Michael D. LaFleur ${ }^{4}$, Kim Lewis, $^{2}$ and Richard E. Lee ${ }^{1 *}$

1. Department of Chemical Biology and Therapeutics, St Jude Children's Research Hospital, 262 Danny Thomas Place, Memphis, TN 38105

2. Antimicrobial Discovery Center, Department of Biology, Northeastern University, 360 Huntington Avenue, Boston, MA 02115

3. Department of Structure Biology, St Jude Children's Research Hospital, 262 Danny Thomas Place, Memphis, TN 38105

4. Arietis Pharma, 650 Albany Street, Suite 114 Boston, Massachusetts 02118

\section{Table of Contents:}

I. Supplementary Figures

- Supplementary Figure 1 Ligand discovery maps...............................................S2

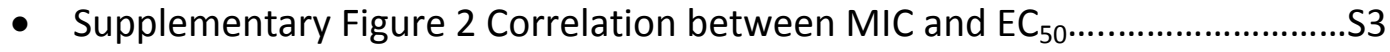

II. Supplementary Tables

- Supplementary Table 1 X-ray Crystallography Data and Refinement S4

- Supplementary Table 2 Minimal inhibitory concentrations......S5

- Supplementary Table 3 Plasma stability.................................................................56

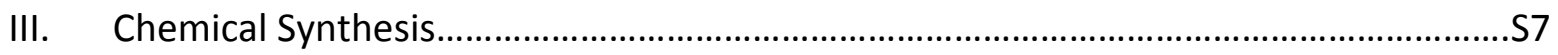

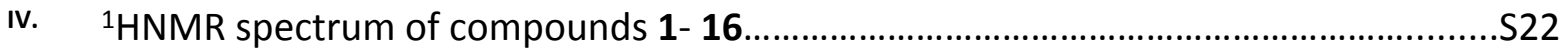

V. Supplementary Table 4 HPLC purity of compounds 1-16.........................................S31 


\section{Supplemental Figures}

A.

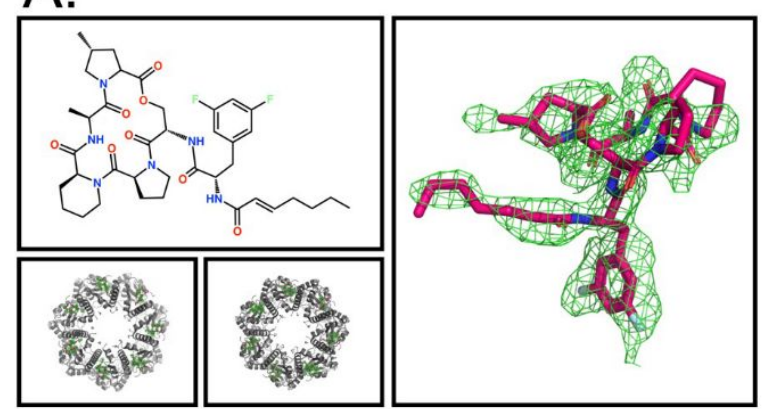

C.

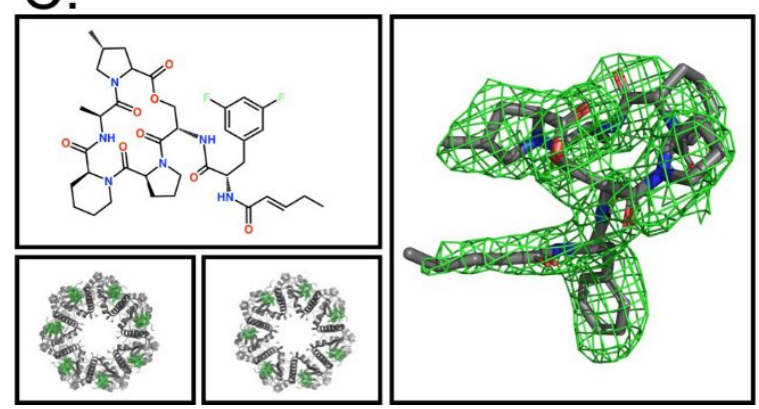

B.
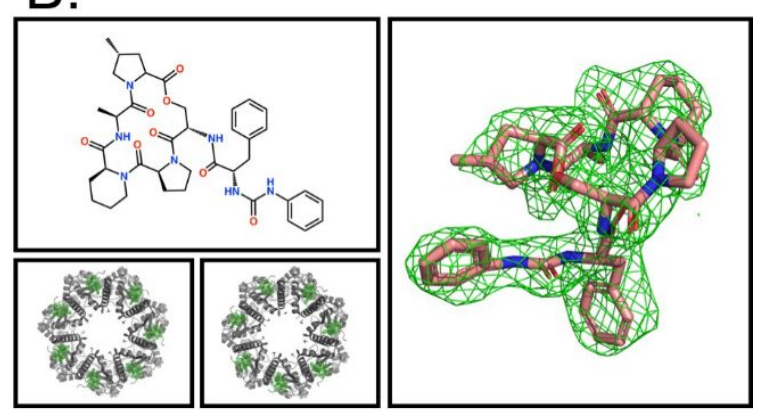

D.
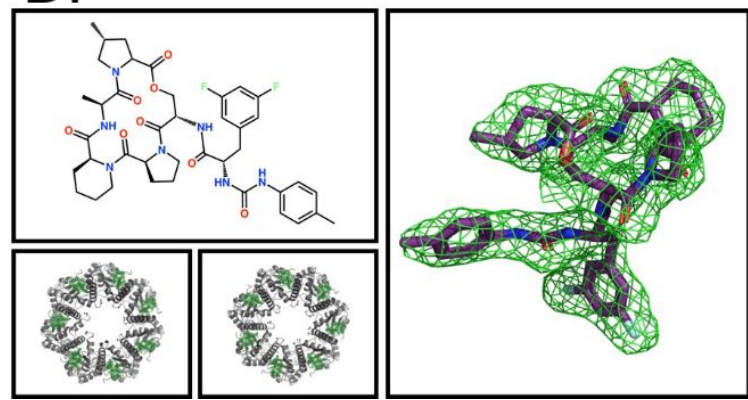

Supplementary Figure 1. Ligand discovery maps. Fo-Fc maps within $2 \AA$ of ligands are shown in green and are contoured at $3 \sigma$. These maps were calculated prior to the addition of ligand into the model. A) ADEP 4 bound to SaClpP PDB: 6PMD. B) Compound 5 bound to SaClpP PDB: 5W18. C) Compound 2 bound to SaClpP PDB: 5VZ2. D) Compound 16 bound to SaClpP PDB: 6PKA. 


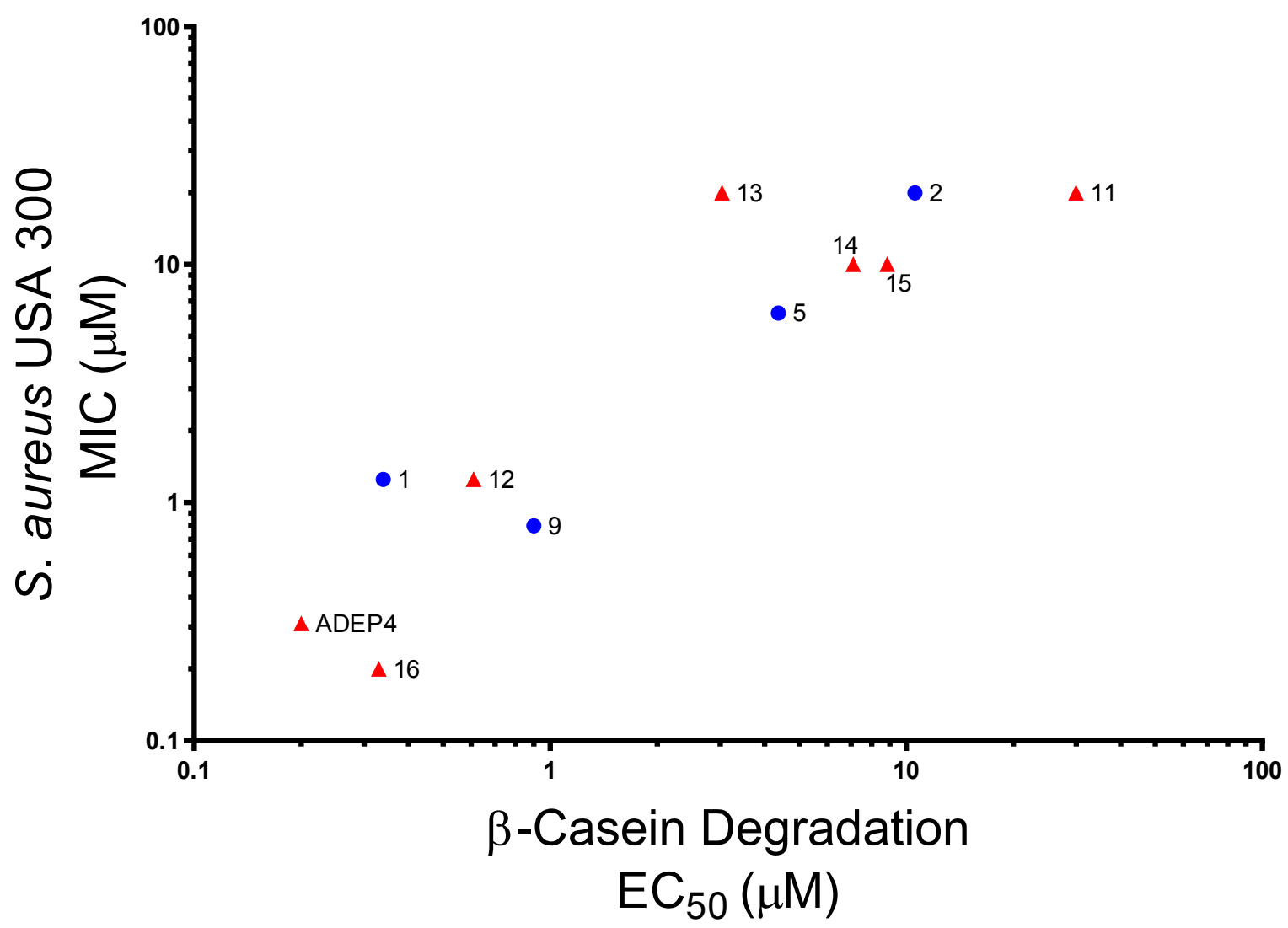

Supplementary Figure 2 . Correlation between $\mathrm{MIC}$ and $\mathrm{EC}_{50}$. Compounds from series 1 are noted with blue circles. Compounds from series 2 are noted with red triangles. 


\section{Supplementary Tables}

\begin{tabular}{|c|c|c|c|c|}
\hline \multicolumn{5}{|l|}{ Supplementary Table 1} \\
\hline Compound & 2 & ADEP4 & 5 & 16 \\
\hline PDB Code & $5 V Z 2$ & 6PMD & $5 W 18$ & 6PKA \\
\hline Wavelength ( $\AA$ ) & 1 & 1 & 1 & 1 \\
\hline Resolution range $(\AA)$ & $2.27-50(2.27-2.35)$ & $2.21-38.77(2.29-2.21)$ & $2.43-50(2.43-2.52)$ & $2.24-40(2.32-2.24)$ \\
\hline Space group & P 1211 & P 1211 & P 1211 & P 1211 \\
\hline Unit cell & $\begin{array}{c}94.56,126.13,145.79 \\
90.00^{\circ} 93.42^{\circ} 90.00^{\circ}\end{array}$ & $\begin{array}{c}94.37,125.97,145.60 \\
90.00 \circ 93.76 \circ 90.00 \circ\end{array}$ & $\begin{array}{c}94.92,126.38,146.06 \\
90.00^{\circ}, 93.42^{\circ}, 90^{\circ}\end{array}$ & $\begin{array}{c}94.96,126.68,146.78 \\
90.00^{\circ}, 93.36^{\circ}, 90\end{array}$ \\
\hline Unique reflections & 155067 (14353) & $1666278(13716)$ & $128084(12694)$ & $160836(15354)$ \\
\hline Multiplicity & $4.0(3.4)$ & $5.2(4.0)$ & $4.0(3.5)$ & $4.2(4.4)$ \\
\hline Completeness (\%) & $97.3(90.4)$ & $98.11(81.40)$ & $99.8(99.3)$ & $97.5(97.8)$ \\
\hline Mean I/sigma(I) & $22.9(3.0)$ & $12.1(2.9)$ & $28.2(5.4)$ & $7.09(2.34)$ \\
\hline Wilson B-factor & 37.63 & 28.14 & 33.44 & 29.0 \\
\hline R-merge & $0.067(0.302)$ & $0.134(0.552)$ & $0.068(0.212)$ & $0.205(0.663)$ \\
\hline Reflections used for R-free & 1996 & & 6556 & $8139(822)$ \\
\hline R-work & $0.1791(0.217)$ & $0.2014(0.2544)$ & $0.1889(0.221)$ & $0.2000(0.2169)$ \\
\hline R-free & $0.2187(0.293)$ & $0.2248(0.2850)$ & $0.2103(0.240)$ & $0.2230(0.2548)$ \\
\hline $\begin{array}{c}\text { Number of non-hydrogen } \\
\text { atoms }\end{array}$ & 20471 & 21307 & 20484 & 20973 \\
\hline Macromolecules & 19078 & 19423 & 19076 & 19075 \\
\hline Ligands & 714 & 717 & 756 & 798 \\
\hline Water & 679 & 1167 & 652 & 1100 \\
\hline RMS(bonds) & 0.018 & 0.011 & 0.025 & 0.010 \\
\hline RMS(angles) & 1.823 & 1.57 & 1.949 & 1.41 \\
\hline
\end{tabular}


Supplementary Table 2. Minimal Inhibitory Concentrations.

Values for Gram positives are shown. Values for the following Gram negative strains were all >200 $\mu \mathrm{M}$ : Klebsiella pneumoniae- ATCC 700603, Acinitobacter baumannii- ATCC 19606, Pseudomonas aeruginosa- ATCC 15692, Escherichia coli- K12, E. coli- K12 $\Delta$ tolC.

\begin{tabular}{|c|c|c|c|c|}
\hline \multicolumn{5}{|c|}{$\begin{array}{l}\text { Minimal Inhibitory Concentration } \\
(\mu \mathrm{M})\end{array}$} \\
\hline & $\begin{array}{l}\text { S. pyogenes } \\
\text { ATCC }\end{array}$ & S. pneumonia & $\begin{array}{l}\text { B. subtilis } \\
\text { ATCC }\end{array}$ & $\begin{array}{l}\text { E.faecalis } \\
\text { ATCC }\end{array}$ \\
\hline Compound & 7000294 & R6 & 23857 & 33186 \\
\hline 1 & $<0.02$ & $<0.02$ & 0.156 & 0.078 \\
\hline ADEP4 & $<0.02$ & 0.04 & 0.313 & 0.078 \\
\hline 2 & 0.625 & 0.63 & 5 & 1.25 \\
\hline 3 & $>20$ & $>20$ & $>20$ & $>20$ \\
\hline 10 & $>20$ & $>20$ & $>20$ & 20 \\
\hline 4 & 20 & 10 & $>20$ & 12.5 \\
\hline 11 & 10 & 10 & 10 & 1.56 \\
\hline 5 & 1.56 & 0.63 & 1.56 & 3.13 \\
\hline 12 & 0.31 & 0.16 & 0.625 & 0.016 \\
\hline 6 & 1.25 & 2.5 & $>20$ & 5 \\
\hline 13 & 0.63 & 0.63 & 12.5 & 0.3 \\
\hline 7 & 3.1 & 0.63 & 6.3 & 6.3 \\
\hline 14 & 1.25 & 1.25 & 5 & 0.31 \\
\hline 8 & 5 & 5 & $>20$ & 20 \\
\hline 15 & 5 & 2.5 & 5 & 0.31 \\
\hline 9 & $<0.2$ & 0.08 & $<0.2$ & $<0.2$ \\
\hline 16 & 0.5 & 0.16 & 2.2 & 0.02 \\
\hline
\end{tabular}


Supplementary Table 3. Plasma Stability

\begin{tabular}{|c|c|c|}
\hline \multicolumn{3}{|c|}{$\begin{array}{c}\text { Plasma Stability } \\
\mathrm{t}_{1 / 2} \text { (hours) }\end{array}$} \\
\hline Compound & Human & Mouse \\
\hline ADEP4 & $>50$ & $>50$ \\
\hline 1 & $>50$ & $>50$ \\
\hline 2 & $>50$ & $29.7 \pm 1.7$ \\
\hline 3 & $>50$ & $24.2 \pm 0.9$ \\
\hline 4 & $>50$ & $>50$ \\
\hline 5 & $>50$ & $15.8 \pm 0.6$ \\
\hline 6 & $>50$ & $>50$ \\
\hline 7 & $>50$ & $>50$ \\
\hline 8 & $>50$ & $>50$ \\
\hline 9 & $>50$ & $33.6 \pm 1.5$ \\
\hline 10 & $>50$ & 31.9 \\
\hline 11 & $>50$ & $>50$ \\
\hline 12 & $>50$ & $>50$ \\
\hline 13 & $>50$ & $>50$ \\
\hline 14 & $>50$ & $>50$ \\
\hline 15 & $>50$ & $>50$ \\
\hline 16 & $>50$ & $35.4 \pm 3.6$ \\
\hline
\end{tabular}




\section{Chemical Synthesis}

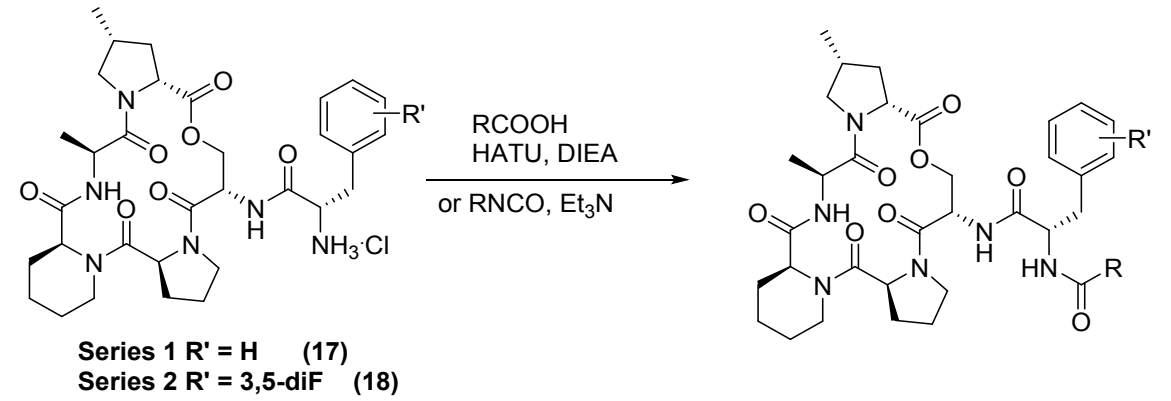

\section{Experimental section}

Starting materials were purchased from commercial sources and were used without further purification. The reactions were monitored by HPLC. The purity of final compounds was determined by UPLC/UV/ELSD/MS. All final compounds' average UV and ELSD purity is $>90 \% .{ }^{10}$ Melting points were obtained on a Thomas Scientific Uni-melt capillary m.p. apparatus (Swedesboro, NJ) and were uncorrected. All ${ }^{1} \mathrm{H}$ spectra were recorded on a Bruker ULTRASHIELD ${ }^{\text {TM }} 400$ plus. The chemical shift values were expressed in ppm (parts per million) relative to tetramethylsilane as internal standard; $\mathrm{s}=$ singlet, $\mathrm{d}=$ double, $\mathrm{t}=$ triplet, $\mathrm{q}=$ quartet, $\mathrm{m}=$ multiplet, br.s $=$ broad singlet. Coupling constants $(\mathrm{J})$ are reported in hertz $(\mathrm{Hz})$.

\section{General synthesis pathway for amide analogs:}

To the solution of compound $\mathbf{1 7 / 1 8}$ in DMF were added added corresponding acid (1.3 eq), HATU (1.3 eq) and DIEA (3.9 eq) at $0^{\circ} \mathrm{C}$, and the reaction mixure was stirred for 18 hours. The reaction solution was diluted with ethyl acetate, washed with a $1 \mathrm{~N}$ hydrochloric acid, aq. saturated sodium hydrogencarbonate solution and aq. saturated sodium chloride solution. The organic phase is dried over sodium sulfate and evaporated. Compound was obtained after reverse phase chlromatography using water/ACN (1:1). 
(E)-N-((S)-1-(((2R,6S,8aS,14aS,20S,23aS)-2,6-dimethyl-5,8,14,19,23-pentaoxooctadecahydro$1 \mathrm{H}, 5 \mathrm{H}, 14 \mathrm{H}, 19 \mathrm{H}-p y r i d o[2,1-i] d i p y r r o l o[2,1-c: 2 ', 1 '-I][1]$ oxa[4,7,10,13]tetraazacyclohexadecin20-yl)amino)-1-oxo-3-phenylpropan-2-yl)hept-2-enamide (1)

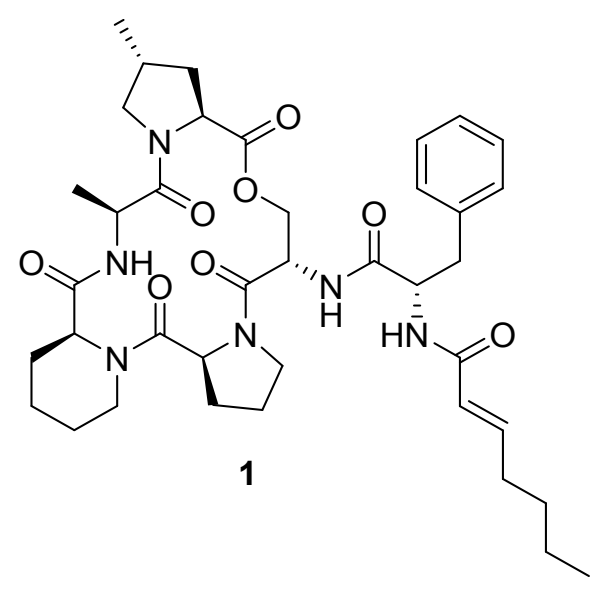

Compound 1 was synthesized from macrocycle 17 (80 mg, $0.11 \mathrm{mmol})$, (E)-hept-2-enoic acid (18.39 mg, $0.14 \mathrm{mmol})$, HATU (54.6 mg, $0.14 \mathrm{mmol})$ and DIEA (75 $\mu \mathrm{L}, 0.43 \mathrm{mmol})$ following general synthesis pathway as a white solid (27.80 mg, $28.5 \%$ ); ${ }^{1} \mathrm{H}$ NMR (400 MHz, Chloroformd) $\delta 0.74(t, J=7.2 \mathrm{~Hz}, 3 \mathrm{H}), 0.86(\mathrm{~d}, \mathrm{~J}=6.6 \mathrm{~Hz}, 3 \mathrm{H}), 1.14-1.24(\mathrm{~m}, 4 \mathrm{H}), 1.27(\mathrm{~d}, \mathrm{~J}=6.6 \mathrm{~Hz}, 3 \mathrm{H})$, $1.57-1.73(\mathrm{~m}, 2 \mathrm{H}), 1.77-1.86(\mathrm{~m}, 2 \mathrm{H}), 1.89-1.96(\mathrm{~m}, 1 \mathrm{H}), 1.98-2.10(\mathrm{~m}, 3 \mathrm{H}), 2.14-2.32(\mathrm{~m}$, $2 \mathrm{H}), 2.45-2.61(\mathrm{~m}, 2 \mathrm{H}), 2.79-2.82(\mathrm{~m}, 2 \mathrm{H}), 2.91-3.04(\mathrm{~m}, 1 \mathrm{H}), 3.31-3.44(\mathrm{~m}, 3 \mathrm{H}), 3.53-$ $3.64(\mathrm{~m}, 2 \mathrm{H}), 4.31-4.39(\mathrm{~m}, 2 \mathrm{H}), 4.41-4.48(\mathrm{~m}, 1 \mathrm{H}), 4.50-4.57(\mathrm{~m}, 2 \mathrm{H}), 4.60-4.67(\mathrm{~m}, 1 \mathrm{H})$, $4.79-4.89(m, 1 H), 4.93-5.00(m, 1 H), 6.00-6.09(m, 1 H), 6.35(d, J=9.6 H z, 1 H), 6.71-6.82$ $(\mathrm{m}, 2 \mathrm{H}), 6.96-7.09(\mathrm{~m}, 3 \mathrm{H}), 7.14(\mathrm{~d}, \mathrm{~J}=7.1 \mathrm{~Hz}, 2 \mathrm{H}), 8.46(\mathrm{~d}, \mathrm{~J}=9.7 \mathrm{~Hz}, 1 \mathrm{H})$. HRMS (ESI) calcd for $\mathrm{C}_{39} \mathrm{H}_{54} \mathrm{~N}_{6} \mathrm{O}_{8}[\mathrm{M}+\mathrm{H}]^{+} 735.4081$, found 735.4068 .

(E)-N-((S)-1-(((2R,6S,8aS,14aS,20S,23aS)-2,6-dimethyl-5,8,14,19,23-pentaoxooctadecahydro1H,5H,14H,19H-pyrido[2,1-i]dipyrrolo[2,1-c:2',1'-I][1]oxa[4,7,10,13]tetraazacyclohexadecin20-yl)amino)-1-oxo-3-phenylpropan-2-yl)pent-2-enamide (2) 


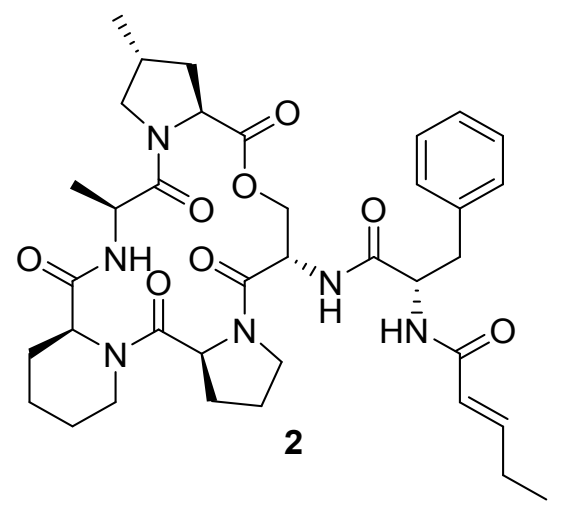

Compound 2 was synthesized from macrocycle 17 (100 mg, $0.14 \mathrm{mmol}$ ), (E)-pent-2-enoic acid (18 $\mathrm{mg}, 0.18 \mathrm{mmol}), \operatorname{HATU}(68.2 \mathrm{mg}, 0.18 \mathrm{mmol})$ and DIEA (94 $\mu \mathrm{L}, 0.54 \mathrm{mmol})$ following general synthesis pathway as a white solid (27.8 mg, $28.5 \%$ ); ${ }^{1} \mathrm{H}$ NMR (400 MHz, Chloroform-d) $\delta 0.99$ $1.11(\mathrm{~m}, 6 \mathrm{H}), 1.40(\mathrm{~d}, \mathrm{~J}=6.5 \mathrm{~Hz}, 3 \mathrm{H}), 1.47-1.53(\mathrm{~m}, 1 \mathrm{H}), 1.65-1.69(\mathrm{~m}, 1 \mathrm{H}), 1.73-1.84(\mathrm{~m}, 2 \mathrm{H})$, $1.94-2.03(\mathrm{~m}, 2 \mathrm{H}), 2.04-2.12(\mathrm{~m}, 1 \mathrm{H}), 2.13-2.30(\mathrm{~m}, 3 \mathrm{H}), 2.31-2.49(\mathrm{~m}, 2 \mathrm{H}), 2.60-2.77(\mathrm{~m}$, $2 \mathrm{H}), 2.90-3.05(\mathrm{~m}, 2 \mathrm{H}), 3.08-3.13(\mathrm{~m}, 1 \mathrm{H}), 3.49-3.63(\mathrm{~m}, 3 \mathrm{H}), 3.70-3.81(\mathrm{~m}, 1 \mathrm{H}), 4.52(\mathrm{~d}, \mathrm{~J}=$ $18.5 \mathrm{~Hz}, 2 \mathrm{H}), 4.67-4.73(\mathrm{~m}, 3 \mathrm{H}), 4.79(\mathrm{~d}, \mathrm{~J}=11.0 \mathrm{~Hz}, 1 \mathrm{H}), 4.97-5.01(\mathrm{~m}, 1 \mathrm{H}), 5.10-5.17(\mathrm{~m}, 1 \mathrm{H})$, $6.22(\mathrm{~d}, \mathrm{~J}=15.4 \mathrm{~Hz}, 1 \mathrm{H}), 6.95-7.01(\mathrm{~m}, 3 \mathrm{H}), 7.12-7.20(\mathrm{~m}, 4 \mathrm{H}), 7.25-7.33(\mathrm{~m}, 3 \mathrm{H}), 8.63(\mathrm{~d}, \mathrm{~J}=9.6$ $\mathrm{Hz}, 1 \mathrm{H}) ; \mathrm{HRMS}(\mathrm{ESI})$ calcd for $\mathrm{C}_{37} \mathrm{H}_{50} \mathrm{~N}_{6} \mathrm{O}_{8}[\mathrm{M}+\mathrm{H}]^{+} 707.3768$, found 707.3773 .

N-((S)-1-(((2R,6S,8aS,14aS,20S,23aS)-2,6-dimethyl-5,8,14,19,23-pentaoxooctadecahydro1H,5H,14H,19H-pyrido[2,1-i]dipyrrolo[2,1-c:2',1'-I][1] oxa[4,7,10,13]tetraazacyclohexadecin20-yl)amino)-1-oxo-3-phenylpropan-2-yl)benzamide (3) 


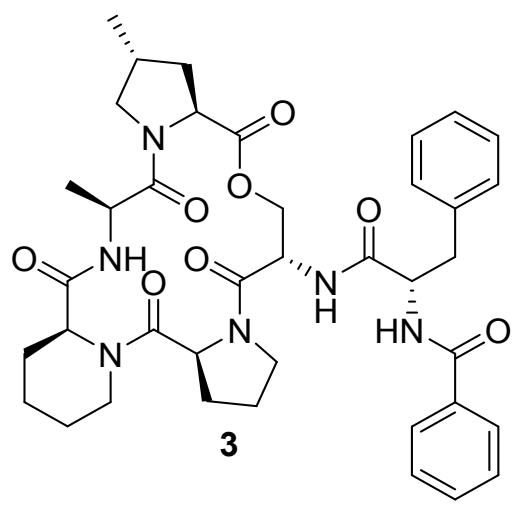

Compound 3 was synthesized from macrocycle $17(70 \mathrm{mg}, 0.097 \mathrm{mmol})$, benzoic acid (15.3 mg, $0.13 \mathrm{mmol})$, HATU $(47.7 \mathrm{mg}, 0.13 \mathrm{mmol})$ and DIEA $(65.8 \mu \mathrm{L}, 0.38 \mathrm{mmol})$ following following general synthesis pathway as a white solid (28 mg, $39.8 \%$ );1H NMR (400 MHz, Chloroform-d) $\delta$ $0.75(\mathrm{~d}, \mathrm{~J}=6.5 \mathrm{~Hz}, 3 \mathrm{H}), 1.32-1.55(\mathrm{~m}, 7 \mathrm{H}), 1.65-1.78(\mathrm{~m}, 4 \mathrm{H}), 1.91-2.04(\mathrm{~m}, 3 \mathrm{H}), 2.05-2.25(\mathrm{~m}$, $2 \mathrm{H}), 2.30-2.40(\mathrm{~m}, 1 \mathrm{H}), 2.50-2.59(\mathrm{~m}, 1 \mathrm{H}), 2.63-2.73(\mathrm{~m}, 2 \mathrm{H}), 2.87-2.92(\mathrm{~m}, 1 \mathrm{H}), 3.05-3.31$ $(m, 3 H), 3.51-3.62(m, 2 H), 3.73-3.82(m, 2 H), 4.46-4.54(m, 2 H), 4.63-4.73(m, 2 H), 4.76-$ $4.88(m, 2 H), 4.91-4.99(m, 1 H), 5.07-5.17(m, 2 H), 7.03(d, J=9.7 \mathrm{~Hz}, 1 \mathrm{H}), 7.41-7.53(\mathrm{~m}, 5 \mathrm{H})$, $7.95-8.00(m, 2 \mathrm{H}), 8.58(\mathrm{~d}, \mathrm{~J}=9.7 \mathrm{~Hz}, 1 \mathrm{H})$; HRMS (ESI) calcd for $\mathrm{C}_{39} \mathrm{H}_{48} \mathrm{~N}_{6} \mathrm{O}_{8}[\mathrm{M}+\mathrm{H}]^{+}$729.3612, found 729.3623 .

(S)-N-((2R,6S,8aS,14aS,20S,23aS)-2,6-dimethyl-5,8,14,19,23-pentaoxooctadecahydro$1 \mathrm{H}, 5 \mathrm{H}, 14 \mathrm{H}, 19 \mathrm{H}-$ pyrido[2,1-i]dipyrrolo[2,1-c:2',1'-I][1] oxa[4,7,10,13]tetraazacyclohexadecin20-yl)-3-phenyl-2-(2-phenylacetamido)propanamide (4) 


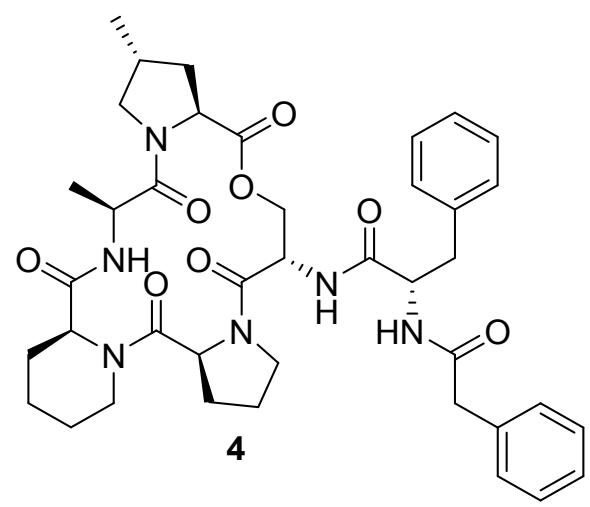

Compound 4 was synthesized from macrocycle 17 ( $27 \mathrm{mg}, 0.043 \mathrm{mmol}$ ), 2-phenylacetic acid (5.88 $\mathrm{mg}, 0.043 \mathrm{mmol})$, HATU (18.1 $\mathrm{mg}, 0.048 \mathrm{mmol})$ and DIEA ( $29.4 \mu \mathrm{L}, 0.17 \mathrm{mmol})$ following general synthesis pathway as a white solid (21.6 mg, 67.3\%); 1H NMR (400 MHz, Chloroform-d) $\delta 1.05$ $(\mathrm{d}, \mathrm{J}=6.6 \mathrm{~Hz}, 3 \mathrm{H}), 1.43(\mathrm{~d}, \mathrm{~J}=6.6 \mathrm{~Hz}, 3 \mathrm{H}), 1.77-1.81(\mathrm{~m}, 1 \mathrm{H}), 1.83-1.91(\mathrm{~m}, 1 \mathrm{H}), 1.93-2.05(\mathrm{~m}$, $2 \mathrm{H}), 2.08-2.13(\mathrm{~m}, 1 \mathrm{H}), 2.15-2.25(\mathrm{~m}, 1 \mathrm{H}), 2.36-2.48(\mathrm{~m}, 2 \mathrm{H}), 2.59-2.69(\mathrm{~m}, 1 \mathrm{H}), 2.73-2.76$ (m, $1 \mathrm{H}), 2.83-3.00(\mathrm{~m}, 2 \mathrm{H}), 3.13-3.21(\mathrm{~m}, 1 \mathrm{H}), 3.49-3.60(\mathrm{~m}, 3 \mathrm{H}), 3.68(\mathrm{~d}, \mathrm{~J}=14.3 \mathrm{~Hz}, 1 \mathrm{H})$, $3.72-3.80(m, 1 H), 3.84(d, J=14.3 \mathrm{~Hz}, 1 \mathrm{H}), 4.44-4.56(\mathrm{~m}, 3 \mathrm{H}), 4.66-4.69(\mathrm{~m}, 2 \mathrm{H}), 4.77-4.84$ $(m, 1 H), 4.97-5.06(m, 1 H), 5.12-5.18(m, 1 H), 6.50(d, J=9.5 \mathrm{~Hz}, 1 \mathrm{H}), 6.98(d, J=7.5 \mathrm{~Hz}, 1 \mathrm{H})$, $7.02-7.07(\mathrm{~m}, 2 \mathrm{H}), 7.18-7.27(\mathrm{~m}, 3 \mathrm{H}), 7.30-7.35(\mathrm{~m}, 2 \mathrm{H}), 7.40-7.45(\mathrm{~m}, 2 \mathrm{H}), 8.57(\mathrm{~d}, \mathrm{~J}=9.6$ $\mathrm{Hz}, 1 \mathrm{H}) ; \mathrm{HRMS}(\mathrm{ESI})$ calcd for $\mathrm{C}_{40} \mathrm{H}_{50} \mathrm{~N}_{6} \mathrm{O}_{8}[\mathrm{M}+\mathrm{H}]^{+} 743.3768$, found 743.3778 .

(S)-N-((2R,6S,8aS,14aS,20S,23aS)-2,6-dimethyl-5,8,14,19,23-pentaoxooctadecahydro$1 \mathrm{H}, 5 \mathrm{H}, 14 \mathrm{H}, 19 \mathrm{H}-$ pyrido[2,1-i]dipyrrolo[2,1-c:2',1'-I][1]oxa[4,7,10,13]tetraazacyclohexadecin20-yl)-3-phenyl-2-(3-phenylpropanamido)propanamide (6) 


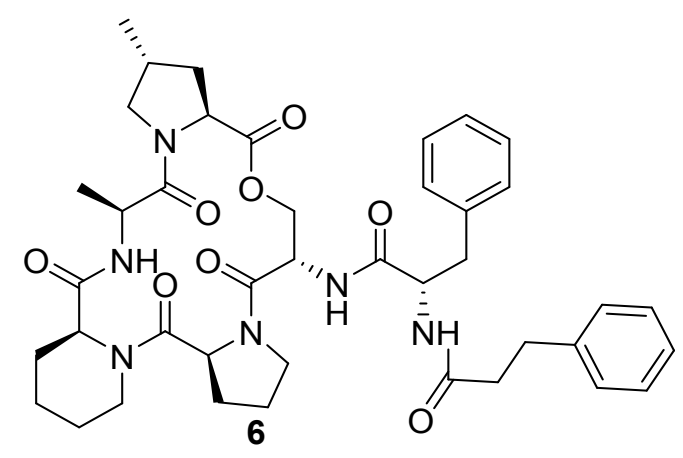

Compound 6 was synthesized from macrocycle 17 (24 mg, $0.038 \mathrm{mmol})$, 3-phenylpropanoic acid (5.77 mg, $0.038 \mathrm{mmol})$, HATU (16.1 mg, $0.042 \mathrm{mmol})$ and DIEA (26.2 $\mu \mathrm{L}, 0.15 \mathrm{mmol})$ following general synthesis pathway as a white solid (17.0 mg, $58.5 \%$ ); $1 \mathrm{H} \mathrm{NMR} \mathrm{(400} \mathrm{MHz,} \mathrm{Chloroform-d)}$ $\delta 1.05(\mathrm{~d}, \mathrm{~J}=6.6 \mathrm{~Hz}, 3 \mathrm{H}), 1.41(\mathrm{~d}, \mathrm{~J}=6.6 \mathrm{~Hz}, 3 \mathrm{H}), 1.67-1.71(\mathrm{~m}, 1 \mathrm{H}), 1.74-1.90(\mathrm{~m}, 2 \mathrm{H}), 1.91-$ $2.04(\mathrm{~m}, 2 \mathrm{H}), 2.06-2.13(\mathrm{~m}, 1 \mathrm{H}), 2.14-2.24(\mathrm{~m}, 1 \mathrm{H}), 2.31-2.52(\mathrm{~m}, 2 \mathrm{H}), 2.60-2.69(\mathrm{~m}, 1 \mathrm{H})$, $2.70-2.78(\mathrm{~m}, 3 \mathrm{H}), 2.79-2.96(\mathrm{~m}, 2 \mathrm{H}), 2.97-3.05(\mathrm{~m}, 2 \mathrm{H}), 3.08-3.19(\mathrm{~m}, 1 \mathrm{H}), 3.45-3.59(\mathrm{~m}$, $3 \mathrm{H}), 3.71-3.80(\mathrm{~m}, 1 \mathrm{H}), 4.45-4.55(\mathrm{~m}, 3 \mathrm{H}), 4.64-4.73(\mathrm{~m}, 2 \mathrm{H}), 4.77-4.83(\mathrm{~m}, 1 \mathrm{H}), 4.94-5.04$ (m, 1H), $5.11-5.17(m, 1 H), 6.42(d, J=9.4 H z, 1 H), 6.90(d, J=7.9 H z, 1 H), 7.07-7.15(m, 2 H)$, $7.16-7.25(\mathrm{~m}, 2 \mathrm{H}), 7.29-7.33(\mathrm{~m}, 5 \mathrm{H}), 8.58(\mathrm{~d}, \mathrm{~J}=9.5 \mathrm{~Hz}, 1 \mathrm{H})$; HRMS (ESI) calcd for $\mathrm{C}_{41} \mathrm{H}_{52} \mathrm{~N}_{6} \mathrm{O}_{8}$ $[M+H]^{+}$757.3925, found 757.3933.

(S)-N-((2R,6S,8aS,14aS,20S,23aS)-2,6-dimethyl-5,8,14,19,23-pentaoxooctadecahydro1H,5H,14H,19H-pyrido[2,1-i]dipyrrolo[2,1-c:2',1'-I][1]oxa[4,7,10,13]tetraazacyclohexadecin20-yl)-3-phenyl-2-(2-(p-tolyl)acetamido)propanamide (8) 


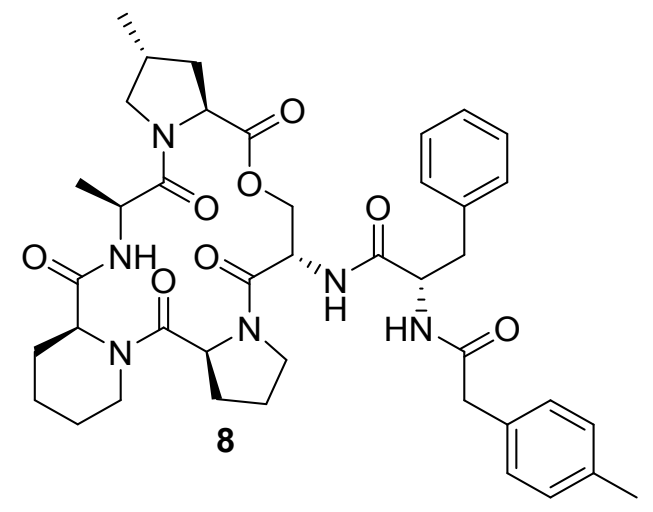

Compound 8 was synthesized from macrocycle 17 (50 mg, $0.069 \mathrm{mmol})$ ) 2-(p-tolyl)acetic acid (10.36 mg, $0.069 \mathrm{mmol})$, HATU (34.1 $\mathrm{mg}, 0.090 \mathrm{mmol}$ ) and DIEA (36.1 $\mu \mathrm{L}, 0.21 \mathrm{mmol}$ ) following general synthesis pathway as a white solid (18.0 mg, 34.5\%); $1 \mathrm{H} \mathrm{NMR} \mathrm{(400} \mathrm{MHz,} \mathrm{Chloroform-d)}$ $\delta 0.97(d, J=6.6 \mathrm{~Hz}, 3 \mathrm{H}), 1.32(\mathrm{~d}, \mathrm{~J}=6.4 \mathrm{~Hz}, 3 \mathrm{H}), 1.58-1.61(\mathrm{~m}, 1 \mathrm{H}), 1.67-1.82(\mathrm{~m}, 2 \mathrm{H}), 1.86-$ $1.95(m, 2 H), 1.99-2.02(m, 1 H), 2.50-2.59(m, 1 H), 2.64-2.68(m, 1 H), 2.78-2.89(m, 5 H)$, $2.94(\mathrm{~s}, 4 \mathrm{H}), 3.02-3.11(\mathrm{~m}, 1 \mathrm{H}), 3.42-3.62(\mathrm{~m}, 4 \mathrm{H}), 3.63-3.80(\mathrm{~m}, 2 \mathrm{H}), 4.38-4.46(\mathrm{~m}, 3 \mathrm{H}), 4.57$ $-4.62(\mathrm{~m}, 2 \mathrm{H}), 4.72(\mathrm{~d}, \mathrm{~J}=11.3 \mathrm{~Hz}, 1 \mathrm{H}), 4.88-4.98(\mathrm{~m}, 1 \mathrm{H}), 5.03-5.11(\mathrm{~m}, 1 \mathrm{H}), 6.58(\mathrm{br} . \mathrm{s}, 1 \mathrm{H})$, $6.93(d, J=7.1 \mathrm{~Hz}, 2 \mathrm{H}), 7.05(\mathrm{~d}, \mathrm{~J}=7.8 \mathrm{~Hz}, 2 \mathrm{H}), 7.07-7.18(\mathrm{~m}, 4 \mathrm{H}), 7.22(\mathrm{~d}, \mathrm{~J}=7.8 \mathrm{~Hz}, 2 \mathrm{H}), 7.98$ (s, $1 \mathrm{H}), 8.45(\mathrm{~d}, \mathrm{~J}=9.4 \mathrm{~Hz}, 1 \mathrm{H})$; HRMS (ESI) calcd for $\mathrm{C}_{41} \mathrm{H}_{52} \mathrm{~N}_{6} \mathrm{O}_{8}[\mathrm{M}+\mathrm{H}]^{+} 757.3925$, found 757.3942.

N-((S)-3-(3,5-difluorophenyl)-1-(((2R,6S,8aS,14aS,20S,23aS)-2,6-dimethyl-5,8,14,19,23pentaoxooctadecahydro-1H,5H,14H,19H-pyrido[2,1-i]dipyrrolo[2,1-c:2',1'I] $[1]$ oxa[4,7,10,13]tetraazacyclohexadecin-20-yl)amino)-1-oxopropan-2-yl)benzamide (10) 
<smiles>C[C@H]1CC(C(=O)OC[C@H](NC(=O)[C@H](Cc2cc(F)cc(F)c2)NC(=O)c2ccccc2)C(=O)N2CC[C@H](O)C2C(=O)N2CCCC[C@H]2C(=O)N[C@@H](C)C(=O)N2C[C@H](C)C[C@H]2C)C(=O)N1</smiles>

Compound 10 was synthesized from macrocycle 18 (90 mg, $0.13 \mathrm{mmol})$, benzoic acid $(15.77 \mathrm{mg}$, $0.13 \mathrm{mmol})$, HATU $(63.8 \mathrm{mg}, 0.17 \mathrm{mmol})$ and DIEA $(67.6 \mu \mathrm{L}, 0.39 \mathrm{mmol})$ following general synthesis pathway as a white solid (12.9 mg, $13.1 \%$ ); $1 \mathrm{H} \mathrm{NMR} \mathrm{(400} \mathrm{MHz,} \mathrm{Chloroform-d)} \delta 0.77$ $(\mathrm{d}, \mathrm{J}=6.6 \mathrm{~Hz}, 3 \mathrm{H}), 1.44(\mathrm{~d}, \mathrm{~J}=6.6 \mathrm{~Hz}, 3 \mathrm{H}), 1.70-1.81(\mathrm{~m}, 2 \mathrm{H}), 1.90-2.04(\mathrm{~m}, 3 \mathrm{H}), 2.06-2.21(\mathrm{~m}$, $2 H), 2.31-2.41(m, 1 H), 2.55-2.66(m, 2 H), 2.66-2.75(m, 1 H), 2.89-2.99(m, 1 H), 3.03-3.18$ $(\mathrm{m}, 2 \mathrm{H}), 3.49-3.65(\mathrm{~m}, 2 \mathrm{H}), 3.74-3.82(\mathrm{~m}, 2 \mathrm{H}), 4.45-4.53(\mathrm{~m}, 2 \mathrm{H}), 4.67-4.71(\mathrm{~m}, 2 \mathrm{H}), 4.75-$ $4.87(m, 2 H), 4.91-5.00(m, 1 H), 5.09-5.16(m, 1 H), 6.63-6.71(m, 1 H), 6.79-6.87(m, 2 H)$, $7.03(\mathrm{~d}, \mathrm{~J}=9.7 \mathrm{~Hz}, 1 \mathrm{H}), 7.42-7.58(\mathrm{~m}, 4 \mathrm{H}), 7.97-8.04(\mathrm{~m}, 2 \mathrm{H}), 8.52(\mathrm{~d}, \mathrm{~J}=9.8 \mathrm{~Hz}, 1 \mathrm{H}) ; \mathrm{HRMS}$ (ESI) calcd for $\mathrm{C}_{39} \mathrm{H}_{46} \mathrm{~F}_{2} \mathrm{~N}_{6} \mathrm{O}_{8}[\mathrm{M}+\mathrm{H}]^{+} 765.3423$, found 765.3430 .

(S)-3-(3,5-difluorophenyl)-N-((2R,6S,8aS,14aS,20S,23aS)-2,6-dimethyl-5,8,14,19,23pentaoxooctadecahydro-1H,5H,14H,19H-pyrido[2,1-i]dipyrrolo[2,1-c:2',1'I][1]oxa[4,7,10,13]tetraazacyclohexadecin-20-yl)-2-(2-phenylacetamido)propanamide (11) 


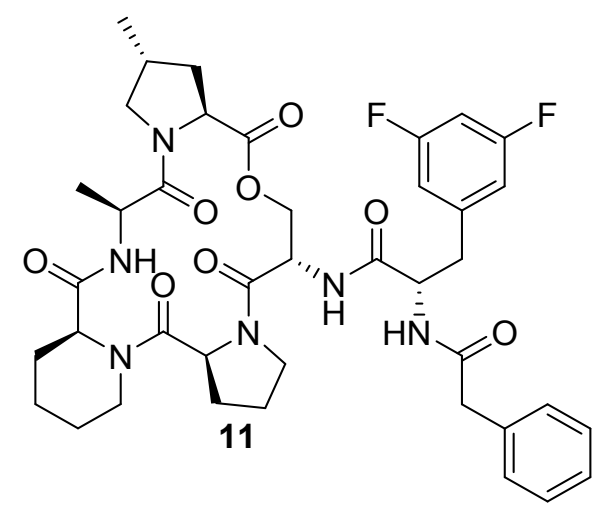

Compound 11 was synthesized from macrocycle 18 (10 mg, $0.015 \mathrm{mmol}), 2$-phenylacetic acid (2.06 mg, $0.015 \mathrm{mmol})$, HATU $(6.33 \mathrm{mg}, 0.017 \mathrm{mmol})$ and DIEA $(10.3 \mu \mathrm{L}, 0.059 \mathrm{mmol})$ following general synthesis pathway as a white solid ( $8.7 \mathrm{mg}, 73.8 \%$ ); $1 \mathrm{H} \mathrm{NMR} \mathrm{(400} \mathrm{MHz,} \mathrm{Chloroform-d)} \delta$ $1.06(\mathrm{~d}, \mathrm{~J}=6.6 \mathrm{~Hz}, 3 \mathrm{H}), 1.39(\mathrm{~d}, \mathrm{~J}=6.6 \mathrm{~Hz}, 4 \mathrm{H}), 1.75-1.91(\mathrm{~m}, 2 \mathrm{H}), 1.93-2.05(\mathrm{~m}, 2 \mathrm{H}), 2.09-$ $2.20(m, 2 H), 2.33-2.52(m, 2 H), 2.56-2.66(m, 1 H), 2.73(d, J=10.1 H z, 1 H), 2.83-2.98(m$, 2H), $3.13-3.21(m, 1 H), 3.51-3.62(m, 3 H), 3.68(d, J=14.2 \mathrm{~Hz}, 1 \mathrm{H}), 3.76-3.86(m, 2 H), 4.46-$ $4.58(m, 3 H), 4.65-4.74(m, 2 H), 4.79-4.85(m, 1 H), 4.96-5.05(m, 1 H), 5.12-5.18(m, 1 H)$, $6.63-6.68(\mathrm{~m}, 3 \mathrm{H}), 6.81(\mathrm{~d}, \mathrm{~J}=9.6 \mathrm{~Hz}, 1 \mathrm{H}), 7.03(\mathrm{~d}, \mathrm{~J}=7.3 \mathrm{~Hz}, 1 \mathrm{H}), 7.31-7.35(\mathrm{~m}, 2 \mathrm{H}), 7.44(\mathrm{~d}, \mathrm{~J}$ $=8.5 \mathrm{~Hz}, 2 \mathrm{H}), 8.48(\mathrm{~d}, \mathrm{~J}=9.6 \mathrm{~Hz}, 1 \mathrm{H})$; HRMS (ESI) calcd for $\mathrm{C}_{40} \mathrm{H}_{48} \mathrm{~F}_{2} \mathrm{~N}_{6} \mathrm{O}_{8}[\mathrm{M}+\mathrm{H}]^{+} 779.3580$, found 779.3584 .

(S)-3-(3,5-difluorophenyl)-N-((2R,6S,8aS,14aS,20S,23aS)-2,6-dimethyl-5,8,14,19,23pentaoxooctadecahydro-1H,5H,14H,19H-pyrido[2,1-i]dipyrrolo[2,1-c:2',1'I][1]oxa[4,7,10,13]tetraazacyclohexadecin-20-yl)-2-(3-phenylpropanamido)propanamide (13) 


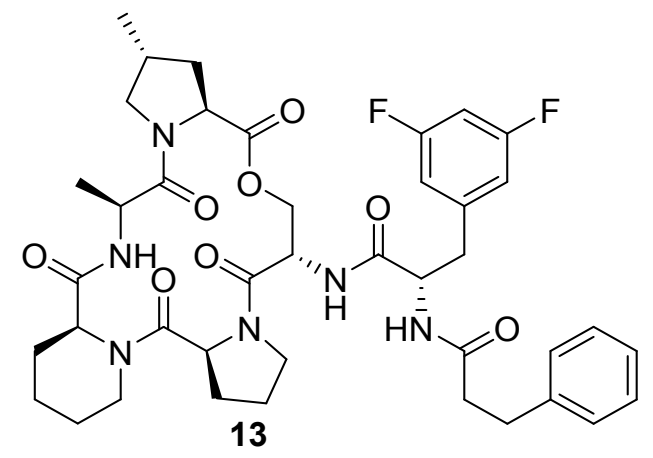

Compound 13 was synthesized from macrocycle 18 (100 mg, $0.15 \mathrm{mmol}$ ), 3-phenylpropanoic acid (29.5 mg, $0.20 \mathrm{mmol}), \operatorname{HATU}(74.8 \mathrm{mg}, 0.197 \mathrm{mmol})$ and DIEA (103 $\mu \mathrm{L}, 0.59 \mathrm{mmol})$ following general synthesis pathway as a white solid (59.1 mg, 49.2 \%); $1 \mathrm{H} \mathrm{NMR} \mathrm{(400} \mathrm{MHz,} \mathrm{Chloroform-d)}$ $\delta 1.03(\mathrm{~d}, \mathrm{~J}=6.6 \mathrm{~Hz}, 3 \mathrm{H}), 1.34(\mathrm{~d}, \mathrm{~J}=6.6 \mathrm{~Hz}, 3 \mathrm{H}), 1.42-1.51(\mathrm{~m}, 2 \mathrm{H}), 1.64-1.72(\mathrm{~m}, 1 \mathrm{H}), 1.79-$ $1.87(\mathrm{~m}, 1 \mathrm{H}), 1.92-2.02(\mathrm{~m}, 2 \mathrm{H}), 2.06-2.15(\mathrm{~m}, 1 \mathrm{H}), 2.30-2.51(\mathrm{~m}, 2 \mathrm{H}), 2.56-2.65(\mathrm{~m}, 1 \mathrm{H})$, $2.67-2.76(m, 2 H), 2.79-2.93(m, 3 H), 2.96-3.05(m, 2 H), 3.07-3.14(m, 1 H), 3.43-3.60(m$, $3 \mathrm{H}), 3.72-3.82(\mathrm{~m}, 1 \mathrm{H}), 4.43-4.57(\mathrm{~m}, 3 \mathrm{H}), 4.68(\mathrm{~d}, \mathrm{~J}=12.4 \mathrm{~Hz}, 2 \mathrm{H}), 4.76-4.81(\mathrm{~m}, 1 \mathrm{H}), 4.91-$ $5.00(\mathrm{~m}, 1 \mathrm{H}), 5.08-5.15(\mathrm{~m}, 1 \mathrm{H}), 6.64-6.70(\mathrm{~m}, 3 \mathrm{H}), 6.79(\mathrm{~d}, \mathrm{~J}=9.6 \mathrm{~Hz}, 1 \mathrm{H}), 6.93(\mathrm{~d}, \mathrm{~J}=7.5 \mathrm{~Hz}$, $1 \mathrm{H}), 7.15-7.22(\mathrm{~m}, 1 \mathrm{H}), 7.27-7.29(\mathrm{~m}, 5 \mathrm{H}), 8.47(\mathrm{~d}, \mathrm{~J}=9.6 \mathrm{~Hz}, 1 \mathrm{H})$; HRMS (ESI) calcd for $\mathrm{C}_{41} \mathrm{H}_{50} \mathrm{~F}_{2} \mathrm{~N}_{6} \mathrm{O}_{8}[\mathrm{M}+\mathrm{H}]^{+} 793.3736$, found 793.3744.

(S)-3-(3,5-difluorophenyl)-N-((2R,6S,8aS,14aS,20S,23aS)-2,6-dimethyl-5,8,14,19,23pentaoxooctadecahydro-1H,5H,14H,19H-pyrido[2,1-i]dipyrrolo[2,1-c:2',1'I] $[1]$ oxa[4,7,10,13]tetraazacyclohexadecin-20-yl)-2-(2-(p-tolyl)acetamido)propanamide (15) 


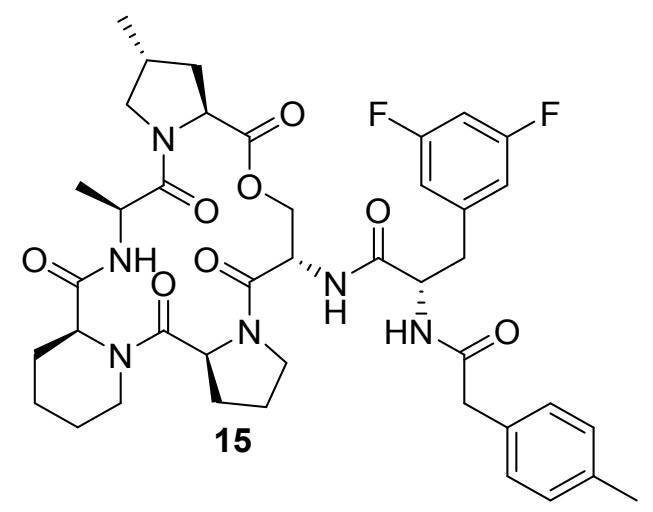

Compound 15 was synthesized from macrocycle 18 (75 mg, $0.11 \mathrm{mmol}$ ), cinnamic acid (17.05 mg, $0.11 \mathrm{mmol})$, HATU $(56.1 \mathrm{mg}, 0.15 \mathrm{mmol})$ and DIEA ( $77 \mu \mathrm{L}, 0.44 \mathrm{mmol})$ following general synthesis pathway as a white solid (36.0 mg, $40.0 \%$ );1H NMR (400 MHz, Chloroform-d) $\delta 8.47$ (d, J = 9.7 $\mathrm{Hz}, 1 \mathrm{H}), 7.31(\mathrm{~d}, \mathrm{~J}=8.0 \mathrm{~Hz}, 2 \mathrm{H}), 7.14(\mathrm{~d}, \mathrm{~J}=7.9 \mathrm{~Hz}, 2 \mathrm{H}), 6.97(\mathrm{~d}, \mathrm{~J}=7.3 \mathrm{~Hz}, 1 \mathrm{H}), 6.71-6.61(\mathrm{~m}$, $3 \mathrm{H}), 6.48(\mathrm{~d}, \mathrm{~J}=9.6 \mathrm{~Hz}, 1 \mathrm{H}), 5.18-5.11(\mathrm{~m}, 1 \mathrm{H}), 5.05-4.96(\mathrm{~m}, 1 \mathrm{H}), 4.85-4.78(\mathrm{~m}, 1 \mathrm{H}), 4.74-$ $4.65(m, 2 H), 4.58-4.42(m, 3 H), 3.83-3.74(m, 2 H), 3.67-3.50(m, 4 H), 3.22-3.13(m, 1 H)$, $2.98-2.82(m, 2 H), 2.77-2.70(m, 1 H), 2.68-2.58(m, 1 H), 2.53-2.31(m, 5 H), 2.23-2.07(m$, $2 \mathrm{H}), 2.05-1.93(\mathrm{~m}, 2 \mathrm{H}), 1.92-1.83(\mathrm{~m}, 1 \mathrm{H}), 1.83-1.76(\mathrm{~m}, 1 \mathrm{H}), 1.71-1.64(\mathrm{~m}, 1 \mathrm{H}), 1.40(\mathrm{~d}, \mathrm{~J}=$ $6.5 \mathrm{~Hz}, 3 \mathrm{H}), 1.07(\mathrm{~d}, \mathrm{~J}=6.6 \mathrm{~Hz}, 3 \mathrm{H})$; HRMS (ESI) calcd for $\mathrm{C}_{41} \mathrm{H}_{50} \mathrm{~F}_{2} \mathrm{~N}_{6} \mathrm{O}_{8}[\mathrm{M}+\mathrm{H}]^{+} 793.3736$, found 793.3753.

\section{General synthesis pathway for urea analogs:}

Corresponding isocyanate ( 1 or 1.2 eq), macrocycle 18 ( 1 eq) and triethylamine (4 or 4.8 eq) were mixed in DCM and the mixture was stirred at room temperature for $10-30 \mathrm{~min}$. The solvents were removed under reduced pressure and the crude residue was purified by reverse-phase flash column chromatography using water to acetonitrile gradient. The fractions containing desired compound were pooled and dried to afford urea analogs. 
(S)-N-((2S,6S,8aS,14aS,20S,23aR)-2,6-dimethyl-5,8,14,19,23-pentaoxooctadecahydro-

$1 \mathrm{H}, 5 \mathrm{H}, 14 \mathrm{H}, 19 \mathrm{H}-$ pyrido[2,1-i]dipyrrolo[2,1-c:2',1'-I][1] oxa[4,7,10,13]tetraazacyclohexadecin-

20-yl)-3-phenyl-2-(3-phenylureido) propanamide (5)

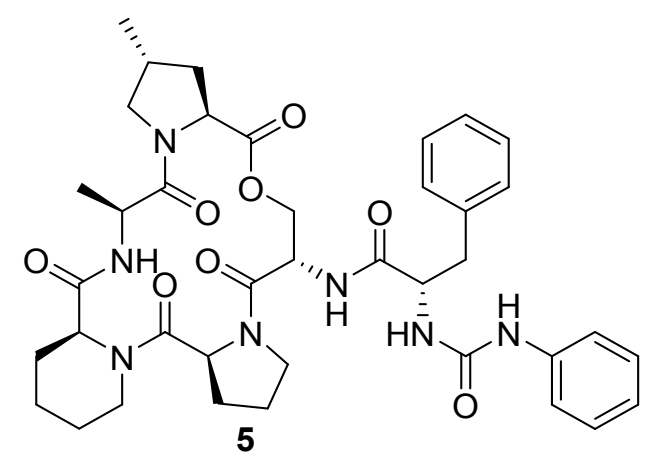

Compound 5 was synthesized from macrocycle $17(61 \mathrm{mg}, 0.097 \mathrm{mmol}$ ), isocyanatobenzene (14 $\mathrm{mg}, 0.12 \mathrm{mmol})$ and triethylamine $(48 \mu \mathrm{L}, 0.35 \mathrm{mmol})$ following general synthesis pathway as a white solid (29.3 mg, 40\%); 1H NMR (400 MHz, Chloroform-d) $\delta 0.93$ (d, J =6.6 Hz, 3H), 1.39 (d, $J=6.6 \mathrm{~Hz}, 3 \mathrm{H}), 1.43-1.58(\mathrm{~m}, 2 \mathrm{H}), 1.75-1.85(\mathrm{~m}, 2 \mathrm{H}), 1.93-2.03(\mathrm{~m}, 2 \mathrm{H}), 2.03-2.12(\mathrm{~m}, 1 \mathrm{H})$, $2.13-2.26(\mathrm{~m}, 1 \mathrm{H}), 2.31-2.46(\mathrm{~m}, 2 \mathrm{H}), 2.59-2.65(\mathrm{~m}, 1 \mathrm{H}), 2.69-2.75(\mathrm{~m}, 1 \mathrm{H}), 2.91-2.96(\mathrm{~m}$, $1 \mathrm{H}), 3.00-3.05(\mathrm{~m}, 1 \mathrm{H}), 3.06-3.15(\mathrm{~m}, 1 \mathrm{H}), 3.45-3.53(\mathrm{~m}, 1 \mathrm{H}), 3.53-3.63(\mathrm{~m}, 2 \mathrm{H}), 3.73-3.79$ (m, 1H), $4.43-4.59(m, 3 H), 4.67-4.72(m, 2 H), 4.79(d, J=11.6 \mathrm{~Hz}, 1 \mathrm{H}), 4.98-5.08(\mathrm{~m}, 1 \mathrm{H})$, 5.12- $5.15(m, 1 H), 5.83(d, J=7.9 \mathrm{~Hz}, 1 \mathrm{H}), 6.80(\mathrm{~d}, \mathrm{~J}=9.6 \mathrm{~Hz}, 1 \mathrm{H}), 6.97(\mathrm{t}, \mathrm{J}=7.4 \mathrm{~Hz}, 1 \mathrm{H}), 7.13-$ $7.26(\mathrm{~m}, 4 \mathrm{H}), 7.27-7.32(\mathrm{~m}, 3 \mathrm{H}), 7.45(\mathrm{~d}, \mathrm{~J}=8.4 \mathrm{~Hz}, 2 \mathrm{H}), 7.88(\mathrm{~s}, 1 \mathrm{H}), 8.68(\mathrm{~d}, \mathrm{~J}=9.6 \mathrm{~Hz}, 1 \mathrm{H})$; 13C NMR (126 MHz, CDCl3) $\delta 172.34,171.41,170.61,169.46,164.55,154.84,139.66,136.02$, $129.52,128.87,128.65,126.83,122.11,119.34,118.33,65.33,60.18,57.29,56.70,55.88$, $54.48,51.13,47.91,46.43,41.32,40.10,38.69,30.95,30.83,29.58,28.11,24.94,23.19,21.37$, 18.12, 18.04; HRMS (ESI) calcd for $\mathrm{C}_{39} \mathrm{H}_{49} \mathrm{~N}_{7} \mathrm{O}_{8}[\mathrm{M}+\mathrm{H}]^{+} 744.3721$, found 744.3726. 
(S)-2-(3-benzylureido)-N-((2R,6S,8aS,14aS,20S,23aS)-2,6-dimethyl-5,8,14,19,23-

pentaoxooctadecahydro-1H,5H,14H,19H-pyrido[2,1-i]dipyrrolo[2,1-c:2',1'-

I][1]oxa[4,7,10,13]tetraazacyclohexadecin-20-yl)-3-phenylpropanamide (7)

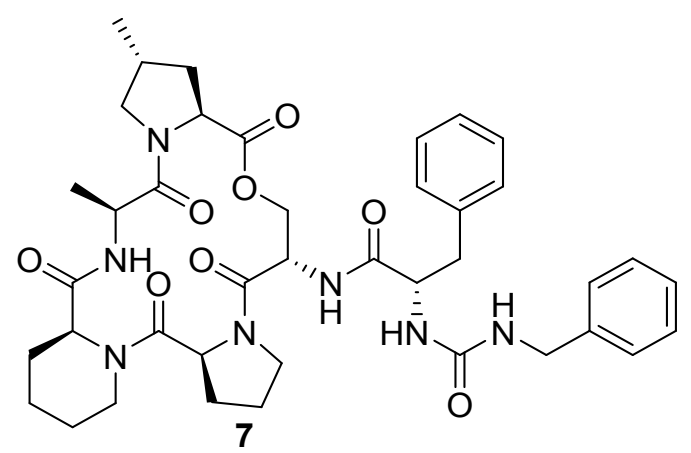

Compound 7 was synthesized from macrocycle 17 (90 mg, $0.14 \mathrm{mmol})$,

(isocyanatomethyl)benzene $(21.75 \mathrm{mg}, 0.16 \mathrm{mmol})$ and triethylamine $(89 \mu \mathrm{L}, 0.65 \mathrm{mmol})$

following general synthesis pathway as a white-off solid (13 mg, $12.6 \%$ ); $1 \mathrm{H} \mathrm{NMR} \mathrm{(400} \mathrm{MHz,}$

Chloroform-d) $\delta 0.94(\mathrm{~d}, \mathrm{~J}=6.6 \mathrm{~Hz}, 3 \mathrm{H}), 1.34(\mathrm{~d}, \mathrm{~J}=6.6 \mathrm{~Hz}, 3 \mathrm{H}), 1.39-1.51(\mathrm{~m}, 2 \mathrm{H}), 1.72-1.81$

$m, 2 H), 1.91-2.01(m, 2 H), 2.02-2.07(m, 1 H), 2.11-2.22(m, 1 H), 2.31-2.41(m, 2 H), 2.59-$

$2.65(m, 1 H), 2.69-2.72(m, 1 H), 2.86-2.93(m, 2 H), 2.97-3.01(m, 1 H), 3.43-3.57(m, 3 H)$,

3.69-3.75 (m, 1H), $4.29-4.41(\mathrm{~m}, 2 \mathrm{H}), 4.44-4.56(\mathrm{~m}, 3 \mathrm{H}), 4.64-4.72(\mathrm{~m}, 2 \mathrm{H}), 4.78(\mathrm{~d}, \mathrm{~J}=11.5$

$H z, 1 H), 4.91-5.01(m, 1 H), 5.10-5.13(m, 1 H), 5.70(d, J=7.9 H z, 1 H), 5.83(t, J=5.8 H z, 1 H)$,

$6.49(\mathrm{~d}, \mathrm{~J}=9.6 \mathrm{~Hz}, 1 \mathrm{H}), 7.12-7.33(\mathrm{~m}, 11 \mathrm{H}), 8.66(\mathrm{~d}, \mathrm{~J}=9.6 \mathrm{~Hz}, 1 \mathrm{H})$; HRMS (ESI) calcd for

$\mathrm{C}_{40} \mathrm{H}_{51} \mathrm{~N}_{7} \mathrm{O}_{8}[\mathrm{M}+\mathrm{H}]^{+} 758.3877$, found 758.3866 .

(S)-N-((2R,6S,8aS,14aS,20S,23aS)-2,6-dimethyl-5,8,14,19,23-pentaoxooctadecahydro-

$1 \mathrm{H}, 5 \mathrm{H}, 14 \mathrm{H}, 19 \mathrm{H}-$ pyrido[2,1-i]dipyrrolo[2,1-c:2',1'-I][1] oxa[4,7,10,13]tetraazacyclohexadecin-

20-yl)-3-phenyl-2-(3-(p-tolyl)ureido)propanamide (9) 


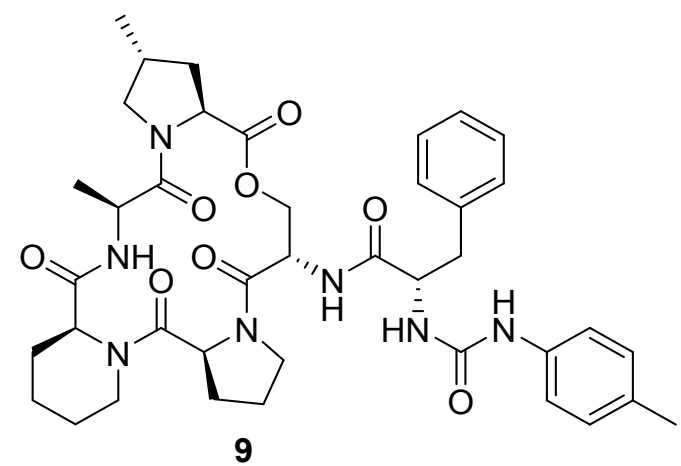

Compound 9 was synthesized from macrocycle 17 (100 mg, 0.15 mmol), 1-isocyanato-4methylbenzene $(24.1 \mathrm{mg}, 0.18 \mathrm{mmol})$ and triethylamine $(99 \mu \mathrm{L}, 0.73 \mathrm{mmol})$ following general synthesis pathway as a white-off solid (34 mg, 30\%); ${ }^{1} \mathrm{H}$ NMR (400 MHz, Chloroform-d) $\delta 0.94$ $(\mathrm{d}, \mathrm{J}=6.5 \mathrm{~Hz}, 3 \mathrm{H}), 1.25(\mathrm{~s}, 3 \mathrm{H}), 1.38(\mathrm{~d}, \mathrm{~J}=6.5 \mathrm{~Hz}, 3 \mathrm{H}), 1.42-1.55(\mathrm{~m}, 2 \mathrm{H}), 1.76-1.85(\mathrm{~m}, 2 \mathrm{H})$, $1.93-2.03(\mathrm{~m}, 2 \mathrm{H}), 2.04-2.09(\mathrm{~m}, 1 \mathrm{H}), 2.13-2.24(\mathrm{~m}, 1 \mathrm{H}), 2.34-2.44(\mathrm{~m}, 2 \mathrm{H}), 2.62(\mathrm{t}, \mathrm{J}=12.4$ $\mathrm{Hz}, 1 \mathrm{H}), 2.71-2.74(\mathrm{~m}, 1 \mathrm{H}), 2.87-2.98(\mathrm{~m}, 1 \mathrm{H}), 3.00-3.04(\mathrm{~m}, 1 \mathrm{H}), 3.06-3.15(\mathrm{~m}, 1 \mathrm{H}), 3.48(\mathrm{t}, \mathrm{J}$ $=10.8 \mathrm{~Hz}, 1 \mathrm{H}), 3.54-3.61(\mathrm{~m}, 2 \mathrm{H}), 3.72-3.78(\mathrm{~m}, 1 \mathrm{H}), 4.41-4.59(\mathrm{~m}, 3 \mathrm{H}), 4.69-4.71(\mathrm{~m}, 2 \mathrm{H})$, $4.79(d, J=11.6 \mathrm{~Hz}, 1 \mathrm{H}), 4.99-5.04(\mathrm{~m}, 1 \mathrm{H}), 5.12-5.14(\mathrm{~m}, 1 \mathrm{H}), 5.80(\mathrm{~d}, \mathrm{~J}=7.9 \mathrm{~Hz}, 1 \mathrm{H}), 6.71(\mathrm{~d}, \mathrm{~J}$ $=9.2 \mathrm{~Hz}, 1 \mathrm{H}), 7.05-7.34(\mathrm{~m}, 9 \mathrm{H}), 7.76(\mathrm{~s}, 1 \mathrm{H}), 8.69(\mathrm{~d}, \mathrm{~J}=9.6 \mathrm{~Hz}, 1 \mathrm{H})$; HRMS (ESI) calcd for $\mathrm{C}_{40} \mathrm{H}_{51} \mathrm{~N}_{7} \mathrm{O}_{8}[\mathrm{M}+\mathrm{H}]^{+} 758.3877$, found 758.3895 .

(S)-3-(3,5-difluorophenyl)-N-((2R,6S,8aS,14aS,20S,23aS)-2,6-dimethyl-5,8,14,19,23pentaoxooctadecahydro-1H,5H,14H,19H-pyrido[2,1-i]dipyrrolo[2,1-c:2',1'I][1]oxa[4,7,10,13]tetraazacyclohexadecin-20-yl)-2-(3-phenylureido)propanamide (12) 


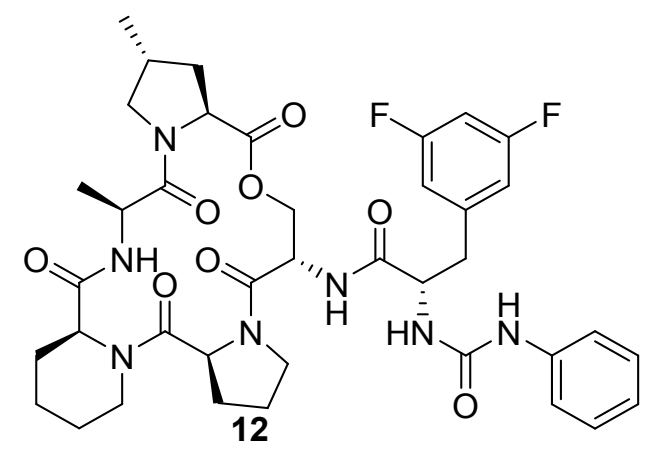

Compound 12 was synthesized from macrocycle $18(61 \mathrm{mg}, 0.097 \mathrm{mmol})$, isocyanatobenzene (14 $\mathrm{mg}, 0.12 \mathrm{mmol}$ ) and triethylamine $(48 \mu \mathrm{L}, 0.35 \mathrm{mmol}$ ) following general synthesis pathway as a white solid (29.3 mg, 40\%); $1 \mathrm{H} \mathrm{NMR} \mathrm{(400} \mathrm{MHz,} \mathrm{Chloroform-d)} \delta 0.96(\mathrm{~d}, \mathrm{~J}=6.5 \mathrm{~Hz}, 3 \mathrm{H}$ ), $1.37(\mathrm{~d}, \mathrm{~J}=6.6 \mathrm{~Hz}, 3 \mathrm{H}), 1.45-1.53(\mathrm{~m}, 2 \mathrm{H}), 1.75-1.91(\mathrm{~m}, 2 \mathrm{H}), 1.96-2.06(\mathrm{~m}, 2 \mathrm{H}), 2.07-2.15$ (m, 1H), $2.15-2.24(m, 1 H), 2.33-2.49(m, 2 H), 2.56-2.67(m, 1 H), 2.72-2.75(m, 1 H), 2.92-$ $3.06(m, 2 H), 3.09-3.19(m, 1 H), 3.50-3.68(m, 3 H), 3.75-3.85(m, 1 H), 4.46-4.61(m, 3 H)$, $4.70-4.74(\mathrm{~m}, 2 \mathrm{H}), 4.84(\mathrm{~d}, \mathrm{~J}=11.6 \mathrm{~Hz}, 1 \mathrm{H}), 4.99-5.09(\mathrm{~m}, 1 \mathrm{H}), 5.12-5.19(\mathrm{~m}, 1 \mathrm{H}), 5.84(\mathrm{~d}, \mathrm{~J}=$ $7.0 \mathrm{~Hz}, 1 \mathrm{H}), 6.65-6.81(\mathrm{~m}, 3 \mathrm{H}), 6.86(\mathrm{~d}, \mathrm{~J}=9.7 \mathrm{~Hz}, 1 \mathrm{H}), 7.01(\mathrm{t}, \mathrm{J}=7.4 \mathrm{~Hz}, 1 \mathrm{H}), 7.31(\mathrm{~d}, \mathrm{~J}=7.5$ $\mathrm{Hz}, 2 \mathrm{H}), 7.47(\mathrm{~d}, \mathrm{~J}=7.7 \mathrm{~Hz}, 2 \mathrm{H}), 7.89(\mathrm{~s}, 1 \mathrm{H}), 8.58(\mathrm{~d}, \mathrm{~J}=9.7 \mathrm{~Hz}, 1 \mathrm{H})$; HRMS (ESI) calcd for $\mathrm{C}_{39} \mathrm{H}_{47} \mathrm{~F}_{2} \mathrm{~N}_{7} \mathrm{O}_{8}[\mathrm{M}+\mathrm{H}]^{+} 780.3532$, found 780.3540 .

(S)-2-(3-benzylureido)-3-(3,5-difluorophenyI)-N-((2R,6S,8aS,14aS,20S,23aS)-2,6-dimethyl5,8,14,19,23-pentaoxooctadecahydro-1H,5H,14H,19H-pyrido[2,1-i]dipyrrolo[2,1-c:2',1'I][1]oxa[4,7,10,13]tetraazacyclohexadecin-20-yl)propanamide (14) 


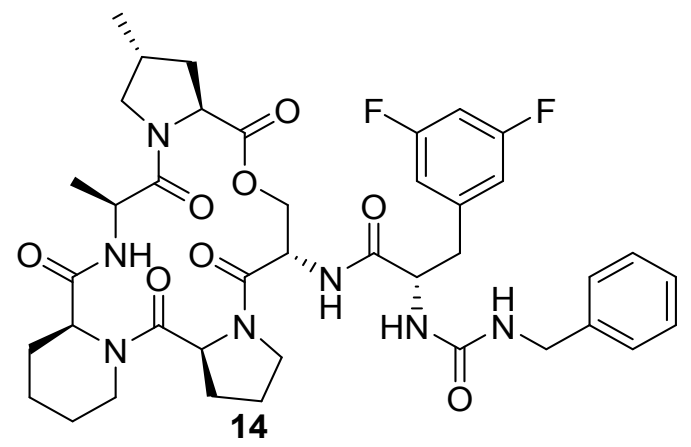

Compound 14 was synthesized from macrocycle $18(80 \mathrm{mg}, 0.12 \mathrm{mmol})$,

(isocyanatomethyl)benzene (16.12 $\mathrm{mg}, 0.12 \mathrm{mmol})$ and triethylamine $(85 \mu \mathrm{L}, 0.48 \mathrm{mmol})$ following general synthesis pathway as a white-off solid (35 mg, 36.4\%); $1 \mathrm{H} \mathrm{NMR} \mathrm{(400} \mathrm{MHz,}$ Chloroform-d) $\delta 0.97(\mathrm{~d}, \mathrm{~J}=6.6 \mathrm{~Hz}, 3 \mathrm{H}), 1.30(\mathrm{~d}, \mathrm{~J}=6.6 \mathrm{~Hz}, 3 \mathrm{H}), 1.44-1.51(\mathrm{~m}, 2 \mathrm{H}), 1.63-1.86$ $(\mathrm{m}, 4 \mathrm{H}), 1.93-2.04(\mathrm{~m}, 2 \mathrm{H}), 2.05-2.11(\mathrm{~m}, 1 \mathrm{H}), 2.13-2.23(\mathrm{~m}, 1 \mathrm{H}), 2.31-2.48(\mathrm{~m}, 2 \mathrm{H}), 2.56-$ $2.62(m, 1 H), 2.70-2.73(m, 1 H), 2.88-2.99(m, 3 H), 3.44-3.53(m, 1 H), 3.54-3.63(m, 2 H)$, $3.74-3.86(m, 1 H), 4.37(d, J=15.0 H z, 1 H), 4.45-4.59(m, 4 H), 4.68-4.72(m, 2 H), 4.79-$ $4.86(m, 1 H), 4.91-5.00(m, 1 H), 5.10-5.18(m, 1 H), 5.70(s, 1 H), 6.64-6.78(m, 3 H), 6.95$ (br.s, $1 \mathrm{H}), 7.22-7.26(\mathrm{~m}, 1 \mathrm{H}), 7.30-7.34(\mathrm{~m}, 4 \mathrm{H}), 8.57(\mathrm{~d}, \mathrm{~J}=9.6 \mathrm{~Hz}, 1 \mathrm{H})$; HRMS (ESI) calcd for $\mathrm{C}_{40} \mathrm{H}_{49} \mathrm{~F}_{2} \mathrm{~N}_{7} \mathrm{O}_{8}[\mathrm{M}+\mathrm{H}]^{+} 794.3689$, found 794.3692.

(S)-3-(3,5-difluorophenyl)-N-((2R,6S,8aS,14aS,20S,23aS)-2,6-dimethyl-5,8,14,19,23pentaoxooctadecahydro-1H,5H,14H,19H-pyrido[2,1-i]dipyrrolo[2,1-c:2',1'I][1]oxa[4,7,10,13]tetraazacyclohexadecin-20-yl)-2-(3-(p-tolyl)ureido)propanamide (16) 


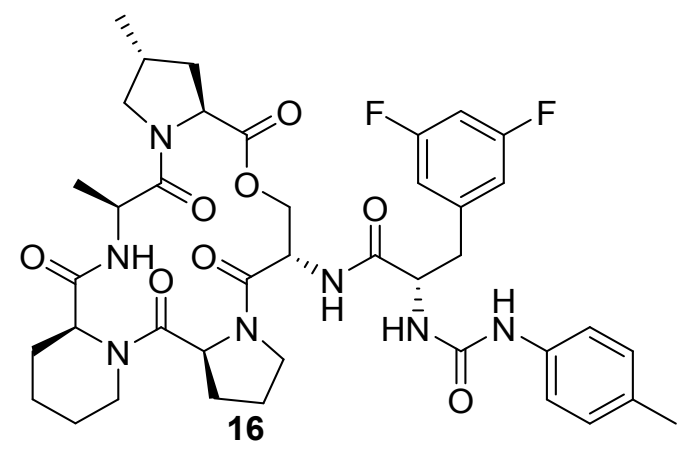

Compound 16 was synthesized from macrocycle $18(100 \mathrm{mg}, 0.14 \mathrm{mmol})$, p-toluene isocyanate (22.9 $\mathrm{mg}, 0.17 \mathrm{mmol}$ ) and triethylamine $(94 \mu \mathrm{L}, 0.69 \mathrm{mmol}$ ) following general synthesis pathway as a white-off solid (34.8 mg, $30.6 \%) ;{ }^{1} \mathrm{H} \mathrm{NMR} \mathrm{(400} \mathrm{MHz,} \mathrm{Chloroform-d)} \delta 0.95$ (d, J = $6.7 \mathrm{~Hz}, 3 \mathrm{H}), 1.33(\mathrm{~d}, \mathrm{~J}=6.6 \mathrm{~Hz}, 3 \mathrm{H}), 1.41-1.51(\mathrm{~m}, 2 \mathrm{H}), 1.60-1.71(\mathrm{~m}, 1 \mathrm{H}), 1.79-1.85(\mathrm{~m}, 2 \mathrm{H})$, $1.93-2.04(\mathrm{~m}, 2 \mathrm{H}), 2.05-2.10(\mathrm{~m}, 1 \mathrm{H}), 2.11-2.23(\mathrm{~m}, 1 \mathrm{H}), 2.28(\mathrm{~s}, 3 \mathrm{H}), 2.35-2.44(\mathrm{~m}, 2 \mathrm{H}), 2.55-$ $2.63(\mathrm{~m}, 1 \mathrm{H}), 2.710-2.72(\mathrm{~m}, 1 \mathrm{H}), 2.90-3.00(\mathrm{~m}, 2 \mathrm{H}), 3.09-3.14(\mathrm{~m}, 1 \mathrm{H}), 3.46-3.67(\mathrm{~m}, 3 \mathrm{H}), 3.74$ - $3.83(\mathrm{~m}, 1 \mathrm{H}), 4.49-4.62(\mathrm{~m}, 3 \mathrm{H}), 4.68-4.72(\mathrm{~m}, 2 \mathrm{H}), 4.81(\mathrm{~d}, \mathrm{~J}=11.5 \mathrm{~Hz}, 1 \mathrm{H}), 4.97-5.05(\mathrm{~m}, 1 \mathrm{H})$, 5.13-5.15 (m, 1H), $5.76(d, J=7.8 \mathrm{~Hz}, 1 \mathrm{H}), 6.61-6.70(\mathrm{~m}, 1 \mathrm{H}), 6.72-6.77(\mathrm{~m}, 2 \mathrm{H}), 7.08(\mathrm{~d}, \mathrm{~J}=8.2$ $\mathrm{Hz}, 2 \mathrm{H}), 7.23(\mathrm{~d}, \mathrm{~J}=9.6 \mathrm{~Hz}, 0.8 \mathrm{H}), 7.33(\mathrm{~d}, \mathrm{~J}=8.4 \mathrm{~Hz}, 2 \mathrm{H}), 7.80(\mathrm{~s}, 1 \mathrm{H}), 8.58(\mathrm{~d}, \mathrm{~J}=9.7 \mathrm{~Hz}, 1 \mathrm{H}) ;{ }^{13} \mathrm{C}$ $\operatorname{NMR}\left(126 \mathrm{MHz}, \mathrm{CDCl}_{3}\right) \delta 172.21,171.75,171.23,170.55,169.54,164.75,163.86,161.98$, $154.92,140.01,136.85,131.73,129.39,118.55,112.70,65.13,60.16,57.22,56.90,55.08$, $54.48,51.23,47.84,46.46,41.19,39.61,38.64,34.68,31.60,30.84,29.61,28.01,25.29,25.02$, $23.15,22.67,21.34,20.73,18.19,17.98,14.14 ; \mathrm{HRMS}$ (ESI) calcd for $\mathrm{C}_{40} \mathrm{H}_{49} \mathrm{~F}_{2} \mathrm{~N}_{7} \mathrm{O}_{8}[\mathrm{M}+\mathrm{H}]^{+}$ 794.3689, found 794.3696. 

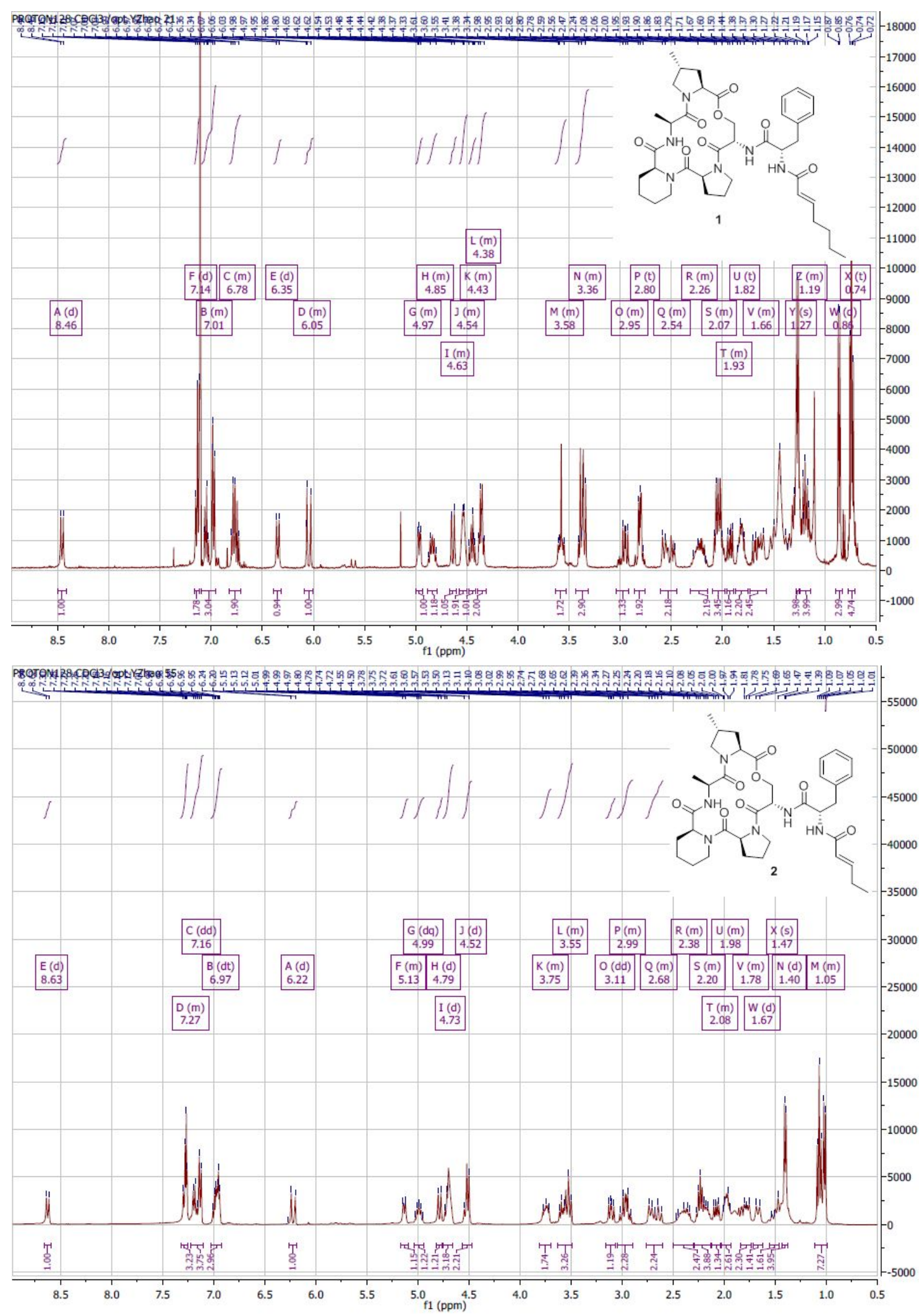

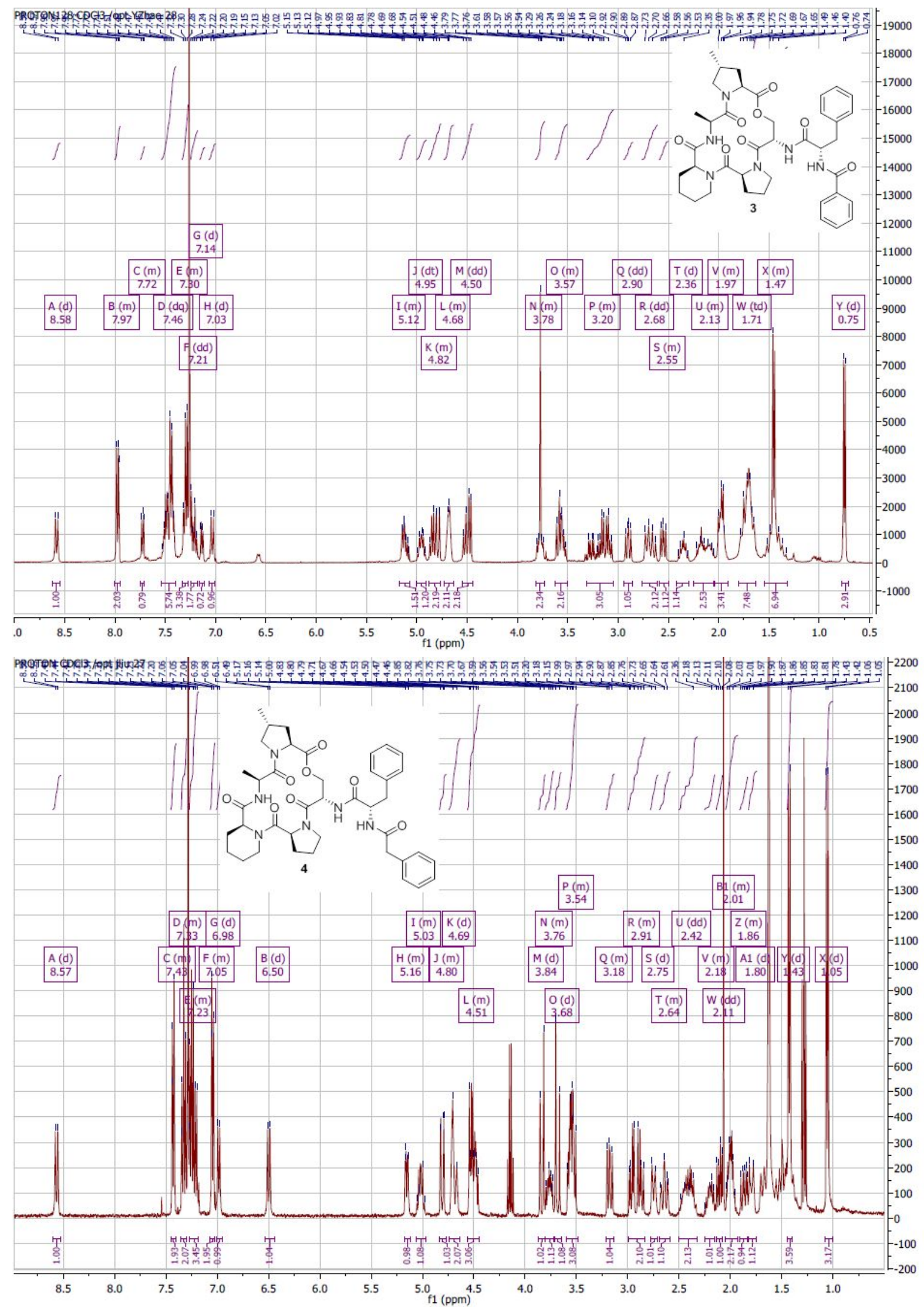


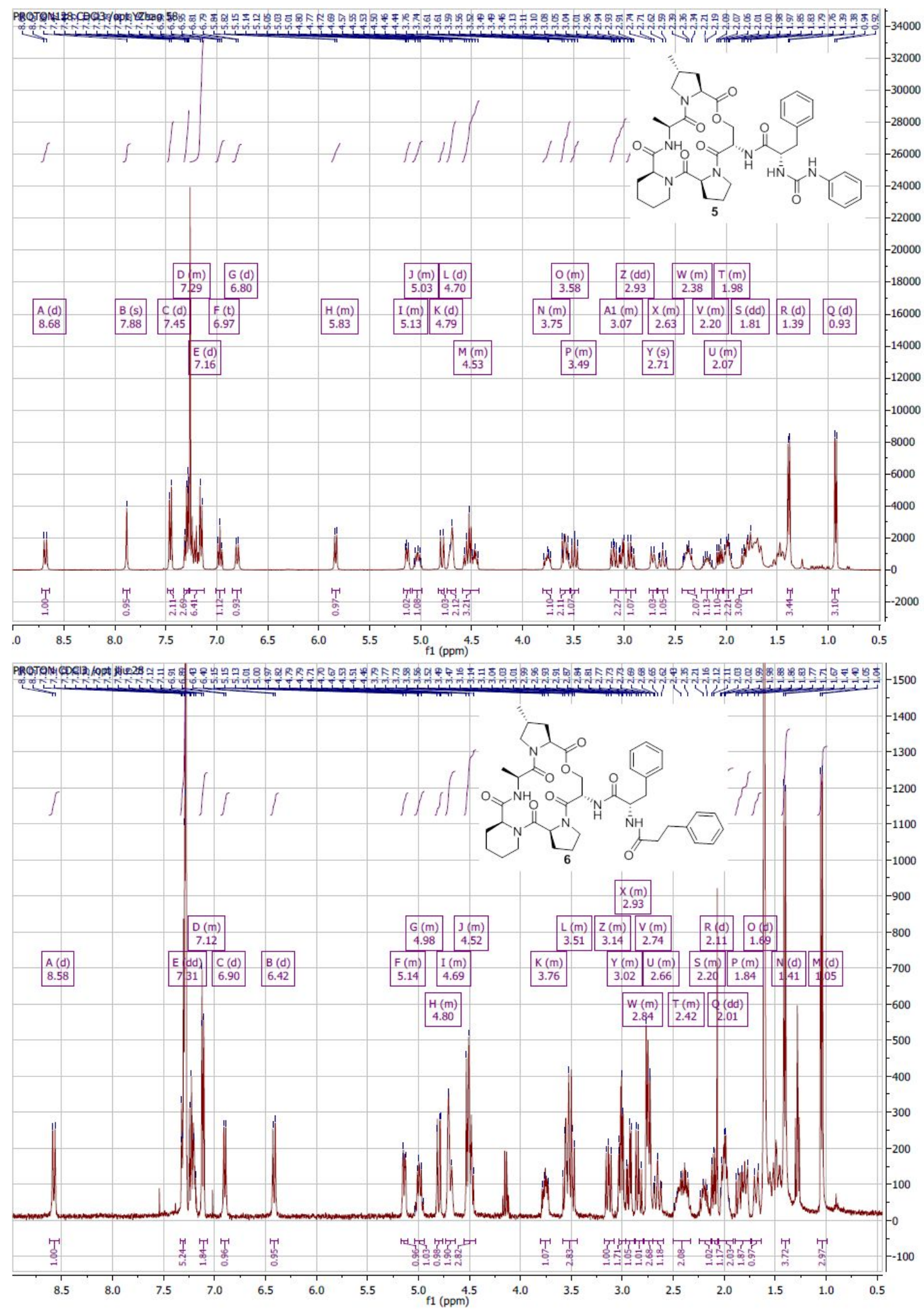




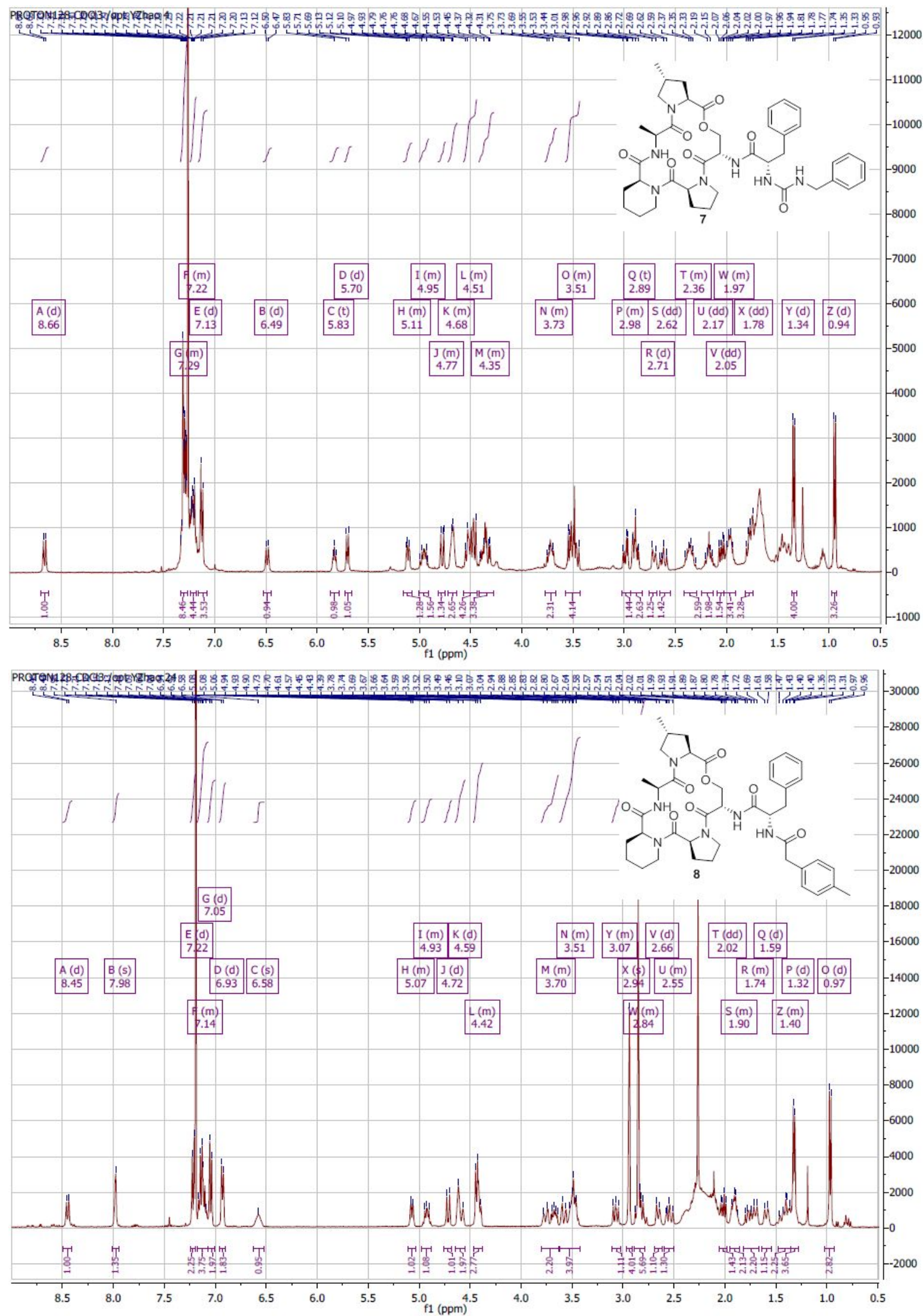



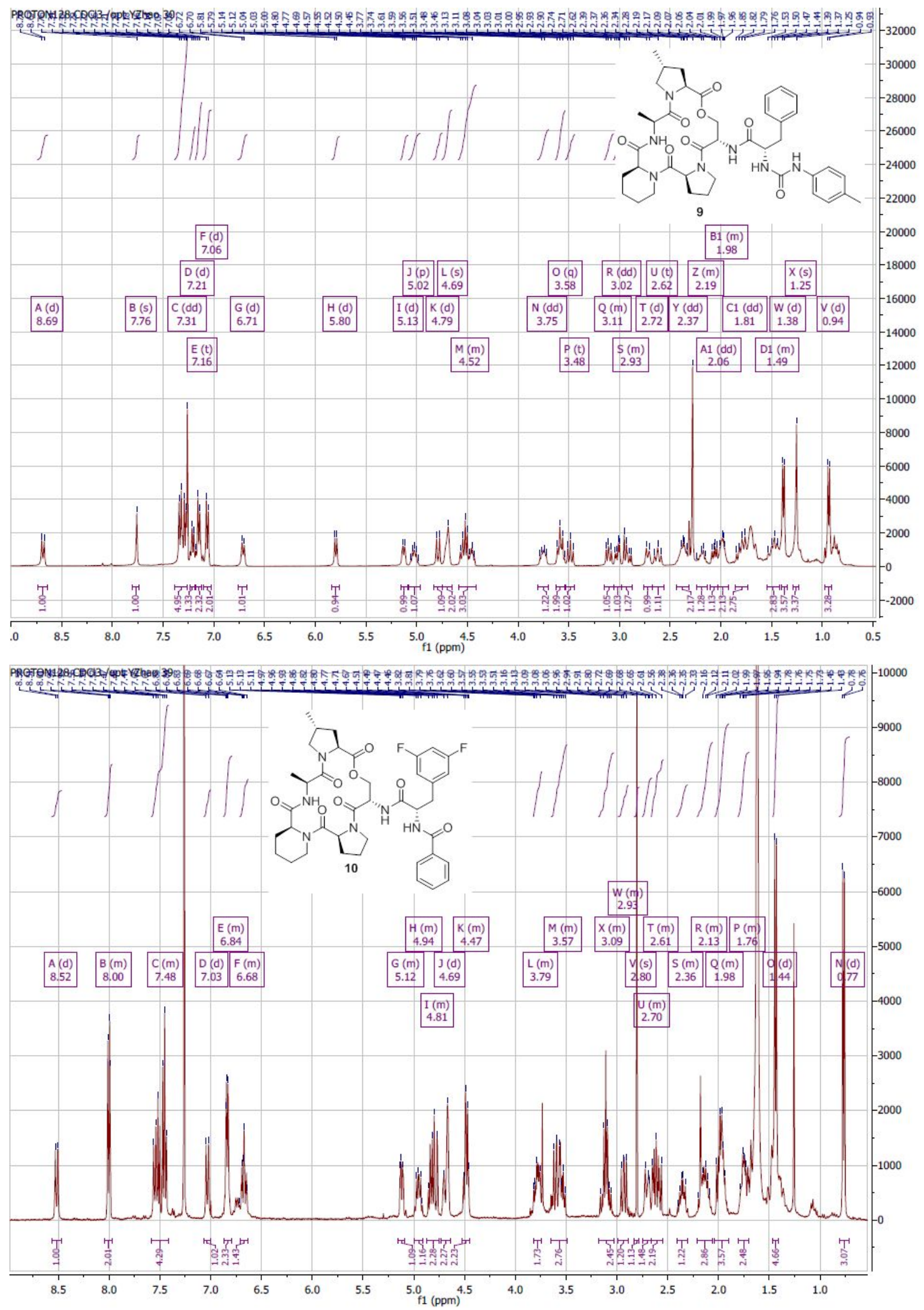

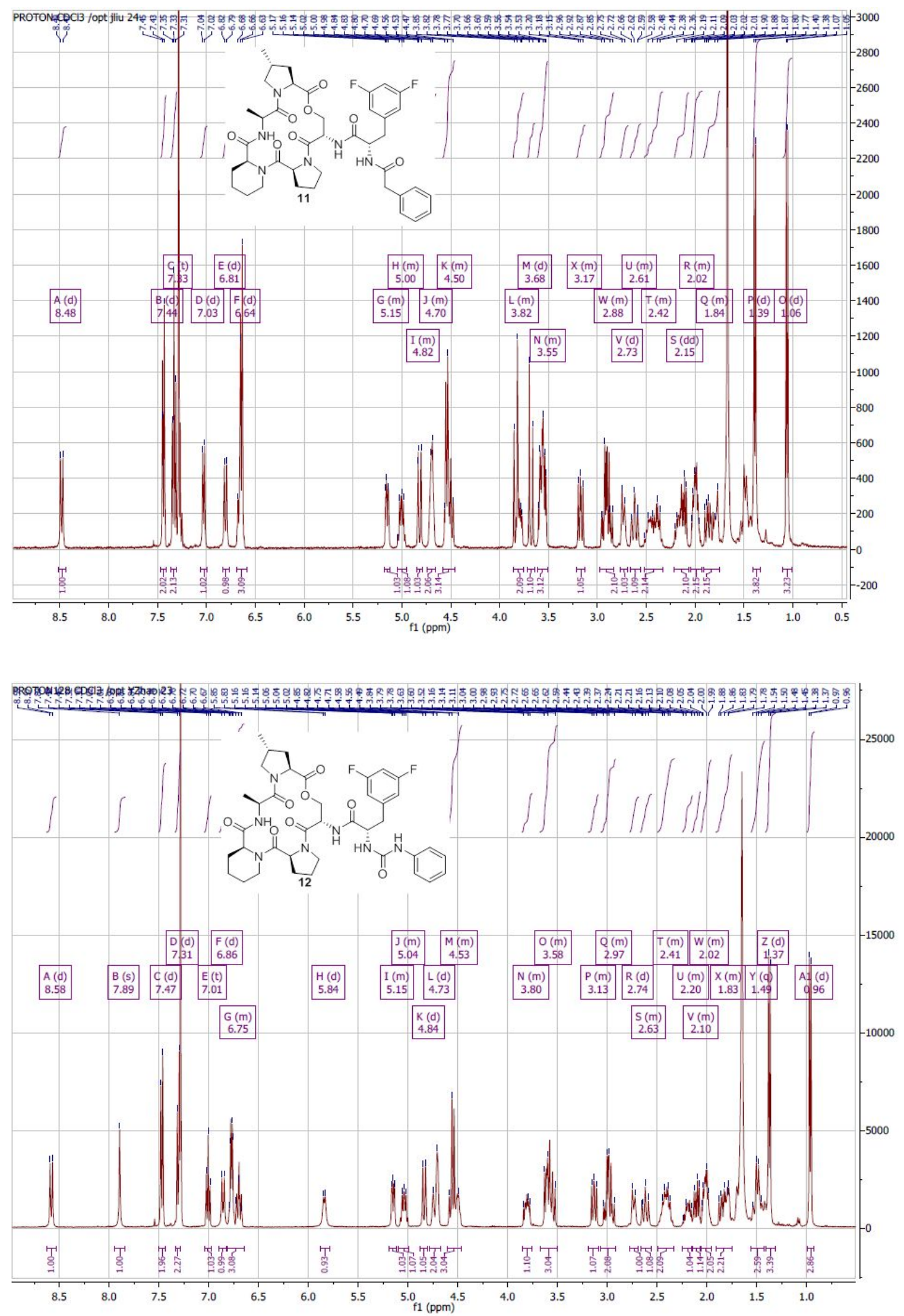

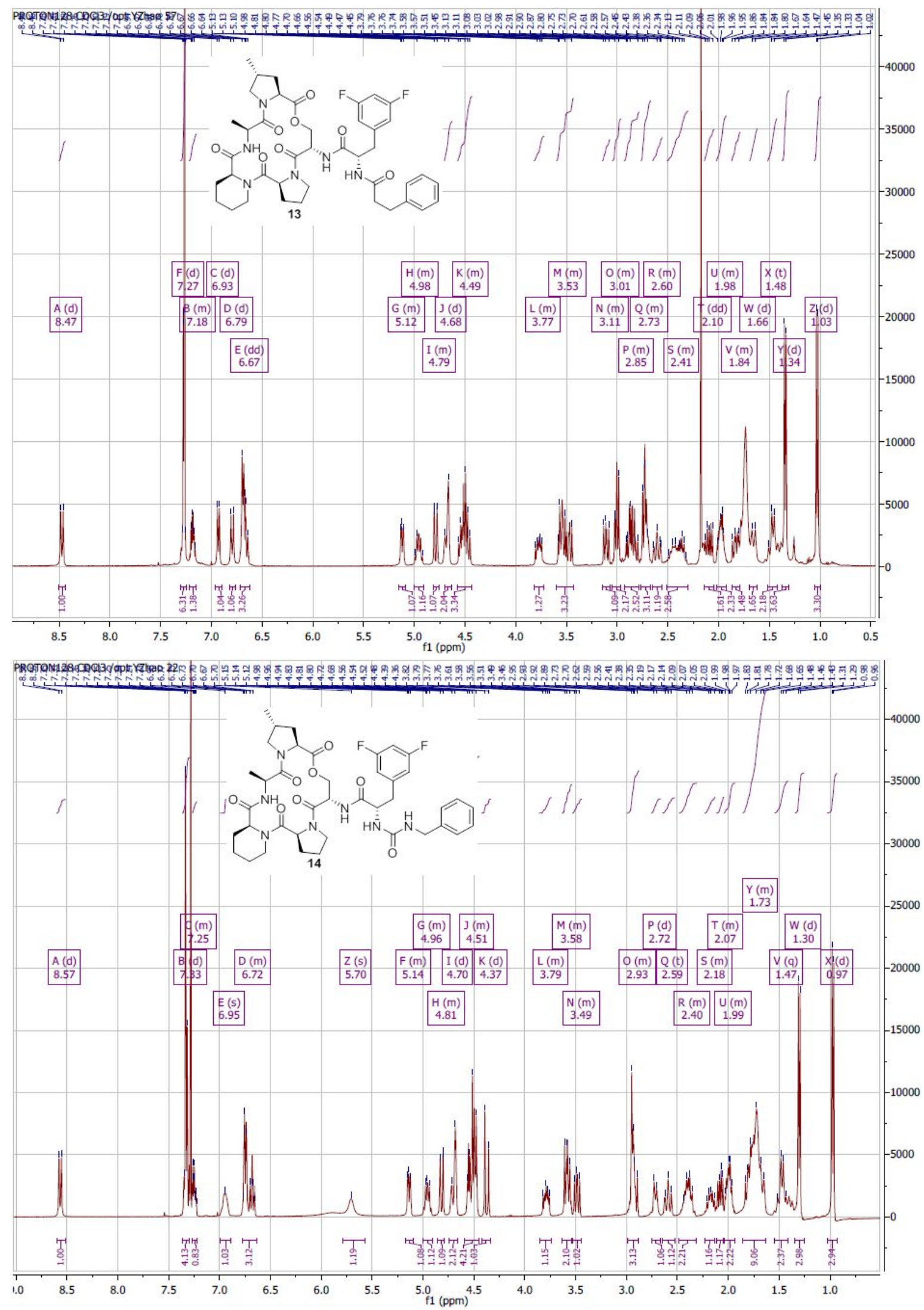

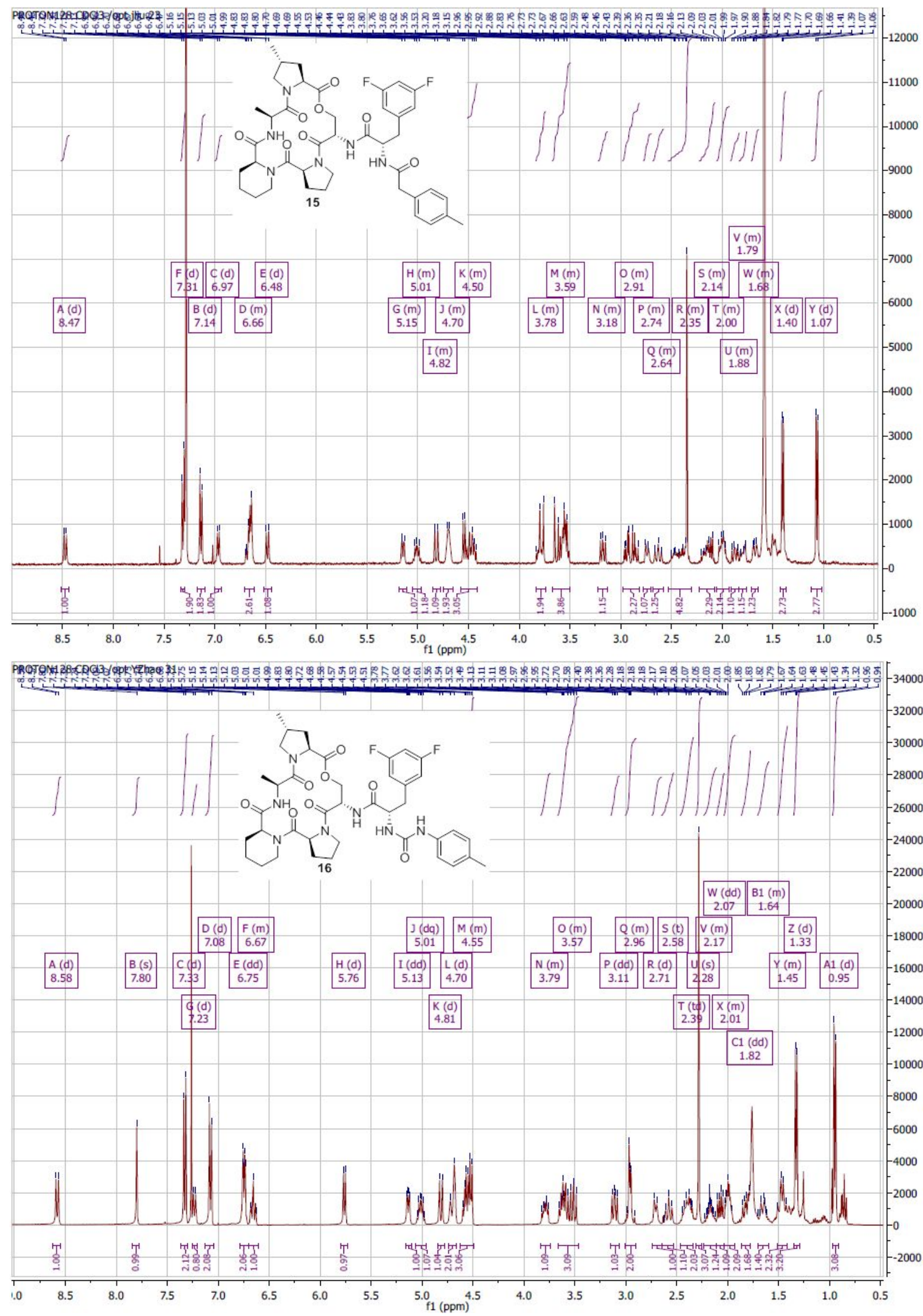
Supplementary Table 4: HPLC purity for final compounds 1 - 16.

\begin{tabular}{|c|c|}
\hline Compound \# & ELSD purity $\%$ \\
\hline $\mathbf{1}$ & 100 \\
\hline $\mathbf{2}$ & 100 \\
\hline $\mathbf{3}$ & 97 \\
\hline $\mathbf{4}$ & 99 \\
\hline $\mathbf{5}$ & 100 \\
\hline $\mathbf{6}$ & 100 \\
\hline $\mathbf{7}$ & 100 \\
\hline $\mathbf{8}$ & 100 \\
\hline $\mathbf{9}$ & 100 \\
\hline $\mathbf{1 0}$ & 100 \\
\hline $\mathbf{1 1}$ & 100 \\
\hline $\mathbf{1 2}$ & 100 \\
\hline $\mathbf{1 3}$ & 100 \\
\hline $\mathbf{1 4}$ & 100 \\
\hline $\mathbf{1 5}$ & 100 \\
\hline $\mathbf{1 6}$ & 100 \\
\hline
\end{tabular}

